\title{
ARTICLE OPEN Group-2 innate lymphoid cell-dependent regulation of tissue neutrophil migration by alternatively activated macrophage-secreted Ear11
}

\author{
Veera Panova ${ }^{1,5}$, Mayuri Gogoi ${ }^{1}$, Noe Rodriguez-Rodriguez ${ }^{1}$, Meera Sivasubramaniam ${ }^{1}$, Helen E. Jolin ${ }^{1}$, Morgan W. D. Heycock ${ }^{1}$, \\ Jennifer A. Walker ${ }^{1}$, Batika M. J. Rana ${ }^{1}$, Lesley F. Drynan ${ }^{1}$, Michael Hodskinson ${ }^{1}$, Richard Pannell ${ }^{1}$, Gareth King ${ }^{1}$, Mark Wing ${ }^{1}$, \\ Andrew J. Easton ${ }^{2}$, Caroline A. Oedekoven ${ }^{3}$, David G. Kent ${ }^{3,6}$, Padraic G. Fallon ${ }^{4}$, Jillian L. Barlow ${ }^{1,6}$ and Andrew N. J. McKenzie (iD ${ }^{1}$
}

Type-2 immunity is characterised by interleukin (IL)-4, IL-5 and IL-13, eosinophilia, mucus production, IgE, and alternatively activated macrophages (AAM). However, despite the lack of neutrophil chemoattractants such as CXCL1, neutrophils, a feature of type-1 immunity, are observed in type- 2 responses. Consequently, alternative mechanisms must exist to ensure that neutrophils can contribute to type-2 immune reactions without escalation of deleterious inflammation. We now demonstrate that type-2 immuneassociated neutrophil infiltration is regulated by the mouse RNase A homologue, eosinophil-associated ribonuclease 11 (Ear11), which is secreted by AAM downstream of IL-25-stimulated ILC2. Transgenic overexpression of Ear11 resulted in tissue neutrophilia, whereas Ear11-deficient mice have fewer resting tissue neutrophils, whilst other type-2 immune responses are not impaired. Notably, administration of recombinant mouse Ear11 increases neutrophil motility and recruitment. Thus, Ear11 helps maintain tissue neutrophils at homoeostasis and during type-2 reactions when chemokine-producing classically activated macrophages are infrequently elicited.

Mucosal Immunology (2021) 14:26-37; https://doi.org/10.1038/s41385-020-0298-2

\section{INTRODUCTION}

The cytokines interleukin (IL)-25 and IL-33 are released rapidly by epithelial cells in the lungs $s^{1,2}$ and intestine ${ }^{3,4}$ in response to type-2 immunity-inducing stimuli, such as allergens and helminthic parasites. These molecules drive type-2 immune responses, promoting the secretion of IL-4, IL-5, IL-9, and IL-13 initially from group 2 innate lymphoid cells (ILC2s), ${ }^{5-7}$ and subsequently from $T$ helper 2 (Th2) cells. ${ }^{8,9}$ This promotes eosinophilia, mucus production, smooth muscle contraction, IgE class switching in B cells, and alternatively activated macrophages (AAM). These responses are required for the robust and timely elimination of parasitic helminth infections in the gut, promoting repair and immune homoeostasis, but also for mediating inappropriate immune activation to allergens. By contrast highly pro-inflammatory pathogen-elicited type-1 immune responses, driven by IFN- $\gamma$ and TLR ligation, activate classically activated macrophages (CAM) leading to their production of inflammatory cytokines, nitric oxide and chemokines such as CXCL1 that recruit neutrophils to sites of microbial infection. However, even during immune homoeostasis and induced type- 2 responses neutrophils are observed without the development of overt type- 1 immunity. This suggests that alternative mechanisms exist to recruit and maintain neutrophils in the absence of type- 1 stimulation.
During type- 2 immunity macrophages respond to IL-4 and IL13 , derived from ILC2s in the innate phase of the response, and express a characteristic alternatively activated profile with high levels of Arginase 1, Relma, Ym-1, and $\mathrm{Ym}-2 .{ }^{10}$ AAMs have been implicated in responses associated with repair and metabolic functions, but also in immune pathology. Interestingly, these AAMs also help maintain immune homoeostasis by counteracting inflammatory immune activation characterised by CAM. ${ }^{10}$ Thus, stimulation of AAM or CAM pathways contribute to maintaining the balance of repair and anti-microbial immunity, and potential immune-mediated pathology.

Although many studies have demonstrated a role for AAMs in tissue remodelling and wound repair, by promoting proliferation and collagen production in fibroblasts, ${ }^{11-13}$ there is evidence that AAMs are also able to perform other functions, including activation of T helper cell subsets and pathogen/host cell killing. ${ }^{14}$ However, since AAMs have been shown to be only weakly phagocytic, ${ }^{15}$ the exact mechanism by which they induce pathogen killing is unclear, and it has been suggested that they also recruit neutrophils to specific tissue sites. ${ }^{10,16}$ Indeed, it has been reported that AAMs and neutrophils collaborate to kill Strongyloides stercoralis in a complement-dependent manner. ${ }^{17}$ In addition, lung macrophages taken from mice 45 days post Nippostrongylus brasiliensis infection,

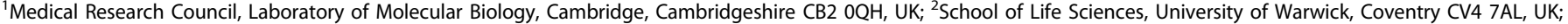
${ }^{3}$ Stem Cell Institute, Clifford-Allbutt Building, Hills Road, Cambridge CB2 OAH, UK and ${ }^{4}$ Trinity Biomedical Sciences Institute, Trinity College Dublin, Dublin, Ireland Correspondence: Jillian L. Barlow (jbarlow@mrc-Imb.cam.ac.uk) or Andrew N. J. McKenzie (anm@mrc-Imb.cam.ac.uk)

${ }^{5}$ Present address: The Francis Crick Institute, London NW1 1AT, UK

${ }^{6}$ Present address: Department of Biology, University of York, Wentworth Way, York YO10 5DD, UK

These authors contributed equally: Mayuri Gogoi, Noe Rodriguez-Rodriguez.

Joint senior authors: Jillian Barlow and Andrew McKenzie.
}

Received: 28 February 2020 Revised: 13 April 2020 Accepted: 22 April 2020

Published online: 26 May 2020 
and transferred to naive animals, promoted parasite clearance from the worm-infected recipients via a mechanism dependent on ' $\mathrm{N} 2$ ' neutrophils (characterised by Arg1, Chi3/3, and Retnla expression). ${ }^{18}$ Neutrophil accumulation, promoted by the chitinases $\mathrm{Ym} 1$ and $\mathrm{Ym} 2$, has also been reported to contribute to $N$. brasiliensis-induced lung injury. ${ }^{19}$ However, the pathways that govern the presence of neutrophils at sites of type-2 inflammation are understudied, and though a number of factors have been proposed, including the chemokine CCL2, ${ }^{20} \mathrm{IL}-5,{ }^{21} \mathrm{IL}-17 \mathrm{~A}$, and $\mathrm{Ym} 1,{ }^{19}$ their mechanism of action, in some cases, remains to be determined.

We observed that intranasal IL-25 treatment, in addition to inducing eosinophilic inflammation also resulted in a concurrent neutrophil infiltration, which coincided with an increase in expression of the mouse RNaseA paralogue, eosinophil-associated ribonuclease 11 (Ear11). Murine eosinophil-associated ribonucleases (Ear) molecules are paralogues of human eosinophil cationic protein (ECP) and eosinophil-derived neurotoxin (EDN), and are associated with asthma. ${ }^{22-24}$ Assays have suggested diverse roles including chemotactic, ${ }^{25}$ bactericidal, ${ }^{26}$ anti-viral, ${ }^{27,28}$, and anti-helminthic ${ }^{29}$ activities in vitro. Notably, transgenic overexpression of Ear11 in mice resulted in augmented neutrophil development in the bone marrow, and neutrophilic infiltrates in the tissues. Furthermore, naive Ear11-deficient mice had fewer neutrophils in the spleen and lungs, and this specific deficit remained following allergen and worm challenge, while other type-2 immune responses were not impaired. IL-25-dependent expression of Ear11 was restricted to AAM, was induced by IL-4 and IL-13 treatment, and was ILC2-dependent, with ILC2-deficient mice (Rora ${ }^{\text {flox/flox}} / 17 \mathrm{raCre}$ ) also showing decreased neutrophilia following IL-25 administration. Mechanistically, administration of recombinant mouse Ear11 (rmEar11) to wild-type mice rapidly recruited neutrophils to the peritoneal cavity. Our data support a novel in vivo role for Ear11 in maintaining tissue neutrophils during homoeostasis and type- 2 reactions when differentiation of classical neutrophil chemoattractant-producing macrophages would not normally be promoted.

\section{RESULTS}

IL-25 induces concomitant tissue eosinophil and neutrophil infiltration in the lungs

Although IL-25 and IL-33 are primarily potent initiators of eosinophilia, ${ }^{30,31}$ we and others have also observed IL-25-induced neutrophil infiltration in the lungs of mice administered with IL-25 intranasally (Fig. 1a)..$^{30,32}$ Time-course analysis also showed a rapid influx of neutrophils in response to IL-33 stimulation (Supplementary Fig. 1a), similar to neutrophil infiltration reported previously in the peritoneal cavity. ${ }^{33}$ Since there is no report of IL-25 receptor expression on neutrophils we performed microarray analysis to study gene expression data from lung tissues exposed to IL-25 and IL-33 to identify potential candidate molecules that could underlie neutrophil infiltration. IL-25 and IL-33 both induced robust upregulation of a number of known type-2 associated molecules, including Clca3, Muc5ac, Chi3/3, Chi3/4, It In 1, and the chemokines Col11, and Ccl17. ${ }^{34}$ In addition, a number of genes with less wellknown type-2 function were also upregulated. One of the most highly upregulated genes was Ear11, ${ }^{34}$ a homologue of RNase A, and a paralogue of ECP and EDN. Human EDN and ECP are induced by type-2 immunity ${ }^{22-24}$ and have been reported to possess chemotactic activities in vitro. ${ }^{25}$ Furthermore, Ear11 has been proposed to act in vitro as a chemoattractant for macrophages. ${ }^{35}$ Although mouse Ear11 expression has been reported in lung alveolar macrophages in models of allergic asthma and following the administration of IL-25 or IL-33, little is known about the functional roles of Ear11 in vivo. To investigate the spatial expression of Ear11 following the induction of type-2 immune responses, tissues were harvested after intranasal administration of either IL-25 or IL-33. Ear11 transcription, determined using quantitative a
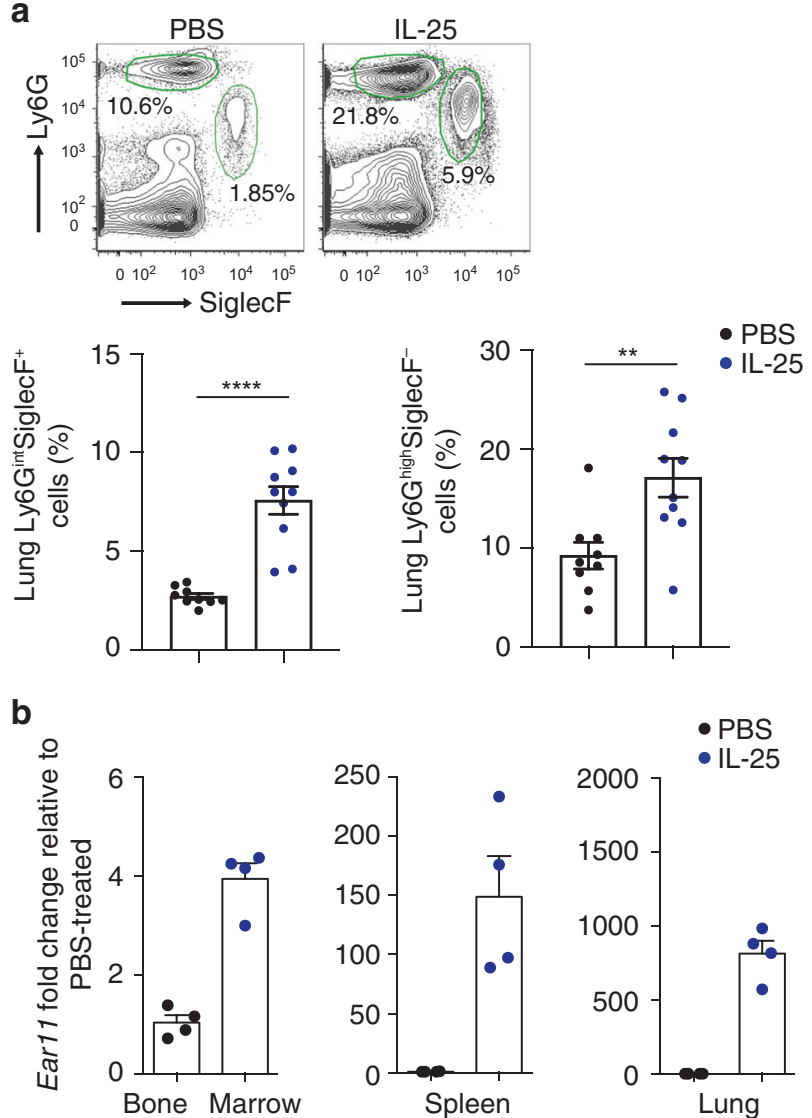

Fig. 1 Ear11 expression is associated with the induction of the type-2 response by IL-25. IL-25 was given intranasally on three consecutive days and lung tissue was analysed. a Flow cytometric analysis of eosinophil (Ly6G ${ }^{\text {int }}$ SiglecF ${ }^{+}$) and neutrophil $\left(\mathrm{Ly}_{6} \mathrm{G}^{\text {high }} \mathrm{Si}\right.$ glecF $^{-}$) infiltration. PBS used as a control. Percentage of cells taken from LiveCD45 ${ }^{+} \mathrm{CD} 11 \mathrm{c}^{-}$gate. Data pooled from two independent experiments $(n=4-5)$. b qPCR determination of Ear11 in bone marrow, spleen, and lung after three doses of IL-25. Data representative of two independent experiments $(n=4)$.

reverse-transcription (RT-PCR), was significantly induced in bone marrow, spleen, and lung, and expression was comparable between IL-25- and IL-33-treated tissue (Fig. 1b and Supplementary Fig. 1b).

Overexpression of Ear11 results in an increased tissue neutrophilia Given the broad tissue distribution of Ear11 gene expression we generated transgenic mice with ubiquitous Ear11 overproduction (Supplementary Fig. 2a). Two founder lines were produced and Ear11 overexpression verified (Supplementary Fig. 2b, c). Strikingly, Ear $11 \mathrm{Tg}$ mice from both lines were noticeably smaller than wild-type littermates (Supplementary Fig. 2d), failed to thrive and did not breed. In addition, post mortem analysis revealed abnormalities in tissue morphology, such as small thymi, enlarged hearts, and gall bladders (Supplementary Fig. 2e). In an effort to circumvent the growth abnormalities observed in the Ear $11 \mathrm{Tg}$ mice and more clearly delineate a role for Ear11 production by haematopoietic cells we generated haematopoietic chimaeras with bone marrow harvested from the Ear11Tg founders (Supplementary Fig. 2f). Sub-lethally irradiated B6SJL mice (CD45.1) were reconstituted with 100\% C57BL/6 (CD45.2) bone marrow (B6SJL:C57BL/6 mice), or Ear11Tg (CD45.2) bone marrow (B6SJL:Ear11Tg mice).

B6SJL:Ear11Tg chimaeric mice displayed elevated Ear11 expression in multiple tissues arising from the transferred bone marrow, and failed to gain weight in the 6 weeks post bone marrow cell 
a

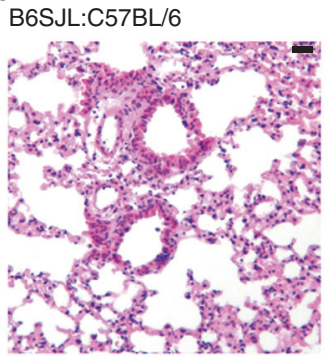

B6SJL:Ear11Tg

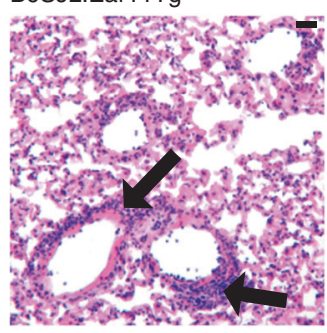

C

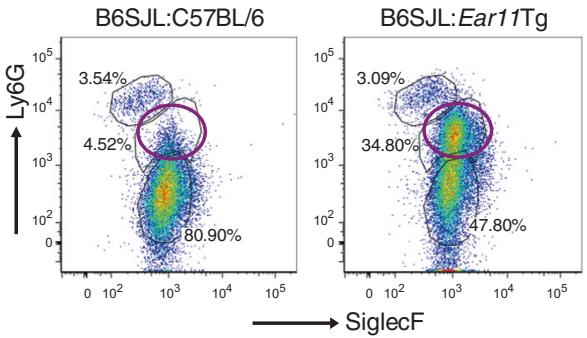

b
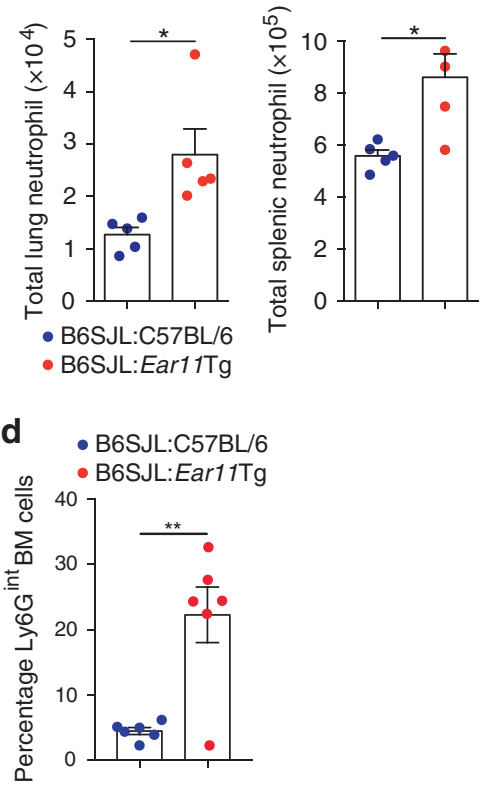

Fig. 2 Transgenic overexpression of Ear11 (Rnase2a) causes neutrophil infiltration. a Inflammation (black arrows) in the lungs of bone marrow chimaeras was analysed in H\&E stained, paraffin-embedded sections ( $\times 20$ magnification, $20 \mu \mathrm{m}$ scale bar). b Neutrophil numbers assessed by flow cytometry in the lung and spleen of bone marrow chimaeras. $\mathbf{c}$, d Flow cytometry plots and graphed data showing analysis of SiglecF and Ly6G expression in bone marrow from chimaeras. Plots are gated on LiveCD45.2 ${ }^{+}$CD $45.1^{-}$CD $11 b^{+}$CD $19^{-}$cells. Open purple circle denotes a previously uncharacterised Ly6Gint bone marrow population shown in the graph (right). Wild-type B6SJL mice received C57BL/ 6 or Ear11Tg bone marrow (BM). Data representative of two independent experiments, $n=4-7$.

reconstitution, as compared with B6SJL:C57BL/6 controls (Supplementary Fig $2 g$, h). Notably, this correlated with neutrophil infiltration in the lung and spleen (Fig. 2a, b), whereas other inflammatory cells, including $T$ cells and eosinophils, were unchanged (Supplementary Fig. 2i-k). Furthermore, although bone marrow eosinophils

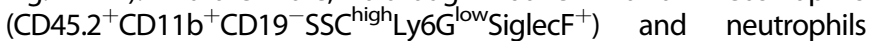
(CD45.2 ${ }^{+}$CD11 b ${ }^{+}$CD 19- ${ }^{-} S C^{\text {high }}$ Ly6G $^{\text {high }}$ SiglecF) ) were identified but unchanged in the B6SJL:C57BL/6, an unexpected population of Ly6G ${ }^{\text {int }}$ SiglecFlo ${ }^{\text {lo }}$ cells was observed in the bone marrow of the B6SJL: Ear11Tg chimaeras (Fig. 2c, d). This was not due to dysfunctional myeloid development, as assessed by colony forming unit analysis (Supplementary Fig. 2l). Taken together these data indicate an unexpected role for haematopoietic cell-derived Ear11 in regulating neutrophilia in vivo.

Ear11 is produced by monocytes, macrophages, and DCs following type-2 provocation in vivo

To examine Ear11-dependent pathways and its function in neutrophil homoeostasis and recruitment, an Ear11 reporter mouse strain was generated by replacing the coding region of the Ear11 gene with a cassette encoding the mCherry fluorescent protein (Supplementary Fig. 3a). The integration of mCherry created a null Ear11 allele with breeding to homozygosity producing an Ear11-deficient mouse (Ear11 Ch/Ch) (Supplementary Fig. 3b). We established the absence of Ear11 transcripts (Supplementary Fig. 3c, d), and corroborated Ear11 protein deficiency in Ear $11^{\mathrm{Ch} / \mathrm{Ch}}$ mice compared with wild-type controls, using antibodies raised against rmEar11 (Supplementary Fig. 4a-e). Pilot studies in naive and type- 2 challenged mice did not show any gross difference in Ear $11 \mathrm{mCherry}$ expression between Ear11 $1^{+/ C h}$ mice and Ear11 ${ }^{\mathrm{Ch} / \mathrm{Ch}}$ mice (data not shown), thus although we cannot completely rule out that absence of Ear11 may alter cell expression patterns the available data do not suggest any abnormality.

In naive Ear $11 \mathrm{mCherry}$ reporter mice, or mice treated with PBS, Ear11 mCherry expression was detected in CD45 ${ }^{+}$Lin (CD3 CD4
CD8 CD19 Gr-1) ${ }^{-}$CD11b ${ }^{+}$Ly6C $^{+}$CD115 ${ }^{+}$monocytes in bone marrow and spleen, and at lower levels in blood monocytes and lung dendritic cells (Fig. 3a, b and Supplementary Fig. 5a-d). Ear11 $1^{+/ C h}$ mice were then treated intraperitoneally or intranasally with PBS or IL-25 to induce a type-2 immune response. IL-25 facilitated a significant increase in Ear $11 \mathrm{mCherry}$ expression in multiple tissues including bone marrow, lung, spleen and blood (Fig. 3b and Supplementary Fig. 5a-d). Ear11mCherry ${ }^{+}$ cells in the bone marrow, spleen and blood conformed to a monocyte phenotype, being $\mathrm{CD}^{+} 5^{+}$Lin (CD3 CD4 CD8 CD19

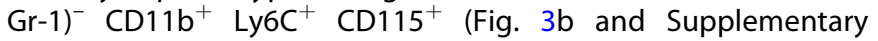
Fig. 5a-d). In the lung, Ear $11 \mathrm{mCherry}$ expression was observed in $\mathrm{CD} 45^{+} \mathrm{CD} 11 \mathrm{C}^{+} \mathrm{F} 4 / 80^{+}$alveolar macrophages and in a population of $\mathrm{CD}_{4} 5^{+} \mathrm{CD} 11 \mathrm{c}^{+} \mathrm{CD} 11 \mathrm{~b}^{+} \mathrm{MHCII}^{\text {high }}$ dendritic cells (Fig. $3 \mathrm{~b}$ and Supplementary Fig. 5d). Confocal microscopy of frozen spleen sections indicated Ear $11 \mathrm{mCherry}^{+}$cells in small clusters that localised to the splenic red pulp, and not to the $T, B$, or marginal zones (Fig. 3c).

To investigate the expression of Ear11 during type-1 and type-2 immune responses Ear $11 \mathrm{mCherry}$ reporter mice were challenged with a number of physiologically relevant immune polarising stimuli. Ragweed pollen (RWP) allergen, when administered intranasally to reporter mice, induced Ear11mCherry in alveolar macrophages (Fig. 3d), localised to the sub-mucosa, away from the basement membrane of the large airways (Fig. 3e). In addition, N. brasiliensis infection or intranasal Alternaria alternata extract challenge induced Ear $11 \mathrm{mCherry}$ expression in lung alveolar macrophages and dendritic cells, respectively (Fig. 3f, Supplementary Fig. 5e, f). By contrast, infection with pneumonia virus of mice (PVM), an enveloped ssRNA - virus that infects lung tissue, and is closely related to human respiratory syncytial virus, failed to induce Ear11mCherry $^{+}$cells (Supplementary Fig. 5g). Similarly, Ear11$\mathrm{mCherry}^{+}$cells were not induced in mice treated intranasally with lipopolysaccharide (LPS), a potent inducer of type-1 immunity (Supplementary Fig. 5h). 
a

Bone marrow gated on $\mathrm{CD} 45^{+}$ Lin $^{-}$CD $11 b^{+}$Ly6C $C^{+}$CD $115^{+}$

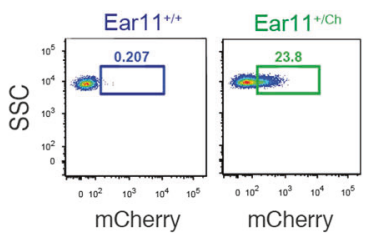

Spleen gated on CD45+ Lin $^{-}$CD $11 b^{+}$Ly6C $C^{+}$CD115

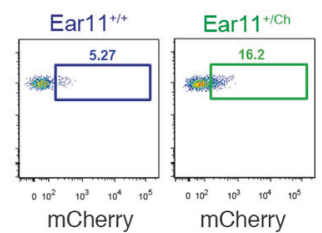

b

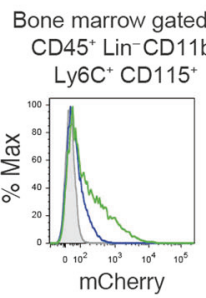

Lung gated on:

D $45^{+} \mathrm{CD} 11 \mathrm{c}^{+} \mathrm{F} 4 / 80$
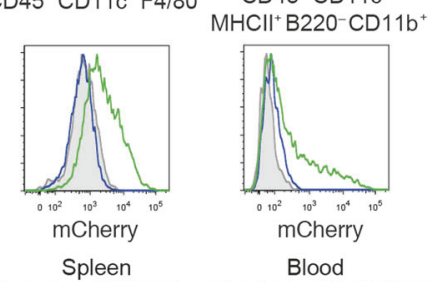

Gated on CD45 $5^{+}$in $^{-}$CD $11 b^{+}$Ly6C C CD $115^{+}$

$$
\begin{aligned}
& \square \text { Ear11 } 1^{+/+} \text {PBS } \\
& \text { - Ear11 } 1^{+/ \mathrm{Ch}} \text { PBS }
\end{aligned}
$$$$
\text { - Ear11 1/Ch IL-25 }
$$
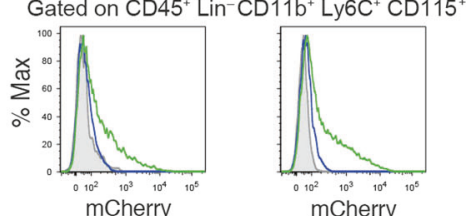

d
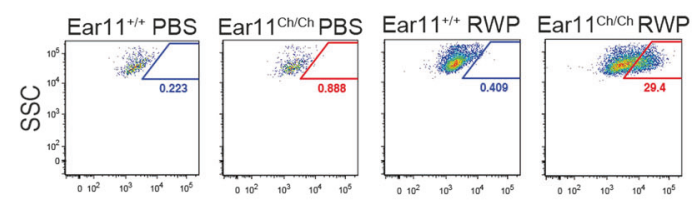

f
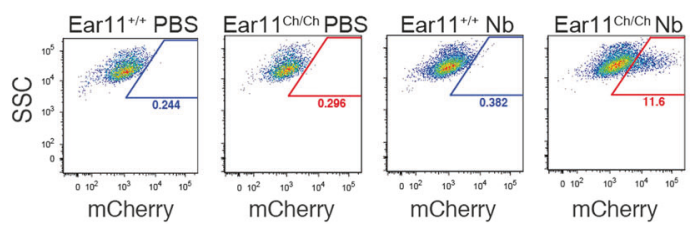
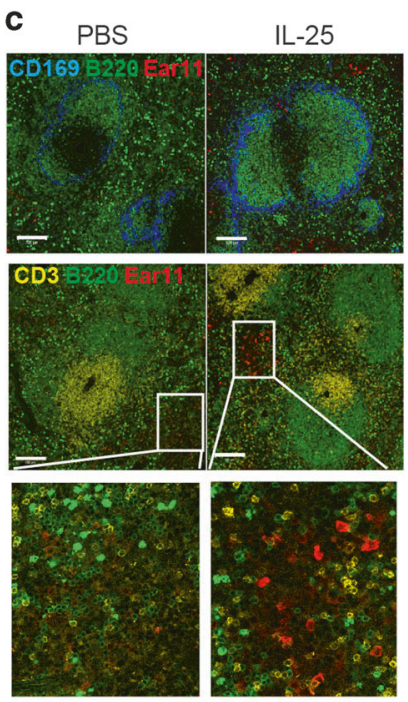

e

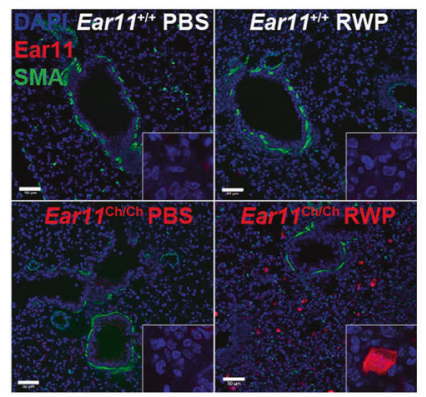

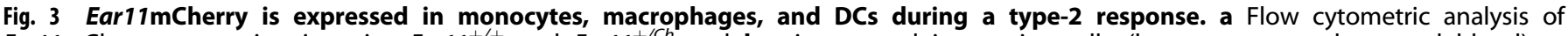

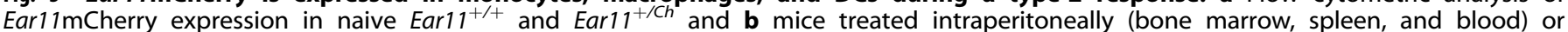
intranasally (lung) as indicated (see Supplementary Fig. 5a-d for gating). Representative of 2-3 independent experiments (with 3-4 mice per experiment). c Confocal microscopy of spleen sections from Ear $11^{+/ C h}$ mice treated intraperitoneally as shown (2 independent experiments,

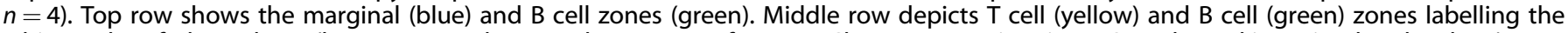
white pulp of the spleen (bottom row shows enlargement of Ear $11 \mathrm{mCherry}$ expression in PBS and cytokine-stimulated spleen). $\times 20$

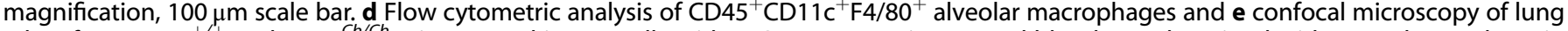
taken from Ear $11^{+/+}$and Ear $11^{\mathrm{Ch} / \mathrm{Ch}}$ mice treated intranasally with PBS or RWP. e Airways and blood vessels stained with smooth muscle actin (SMA, green); all nuclei were counterstained with DAPI (blue). Two independent experiments, $n=5-8$; $\times 20$ magnification with $\times 63$ inset,

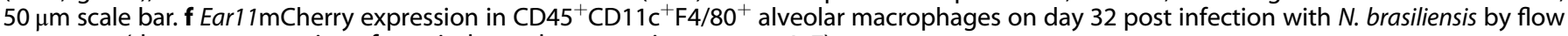
cytometry (data representative of two independent experiments, $n=4-7$ ).

Together, these data indicate that Ear11 expression is upregulated during type- 2 immunity, but not type- 1 immune responses, and is restricted to the myeloid lineage.

\section{IL-4, IL-13, and ILC2 are required for Ear11 expression by} alternatively activated macrophages

The association of Ear11 expression with myeloid cells in type-2 immunity specifically raised the possibility that Ear11 may represent a product of AAMs that arises in response to type-2 cytokine stimulation. Relma and Arginase 1 are markers of AAM. ${ }^{36}$ Both IL-25 and IL-33 induced Relma and Arginase 1 expression by lung alveolar macrophages (Supplementary Fig. 6a). IL-25-induced Ear $11 \mathrm{mCherry}$-positive and -negative alveolar macrophages were isolated and assessed for markers of alternative activation. The
Ear $11 \mathrm{mCherry}{ }^{+}$macrophages were highly restricted to the Retnla (Relma)-positive, Arg1-positive AAM population (Fig. 4a). Similarly, IL-33 induced Ear $11 \mathrm{mCherry}{ }^{+}$macrophages also showed higher expression of AAM signature genes (Supplementary Fig. 6b, c).

To understand whether IL-25 could directly induce Ear11 expression, or if this was mediated by cytokines downstream of IL-25, such as IL-4 and IL-13, peritoneal macrophages were purified from naive Ear $11^{+/ C h}$ mice and cultured with media or the recombinant cytokines IL-25, IL-33, IL-13, and IL-4. IL-25 and IL-33 did not induce Ear11, whilst only a modest induction was caused by IL-13, as assessed by Ear $11 \mathrm{mCherry}$ production (Fig. 4b), the detection of Ear11 mRNA (Fig. 4c), and Ear11 protein expression (Fig. 4d). By contrast, treatment with IL-4 rapidly upregulated Ear11 (Fig. 4b-d). Ear11 expression by 

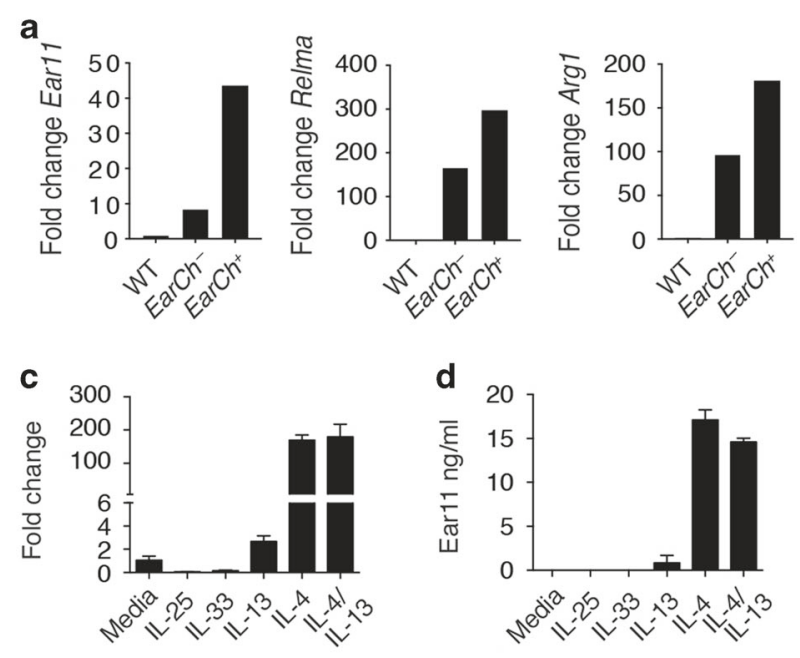

e

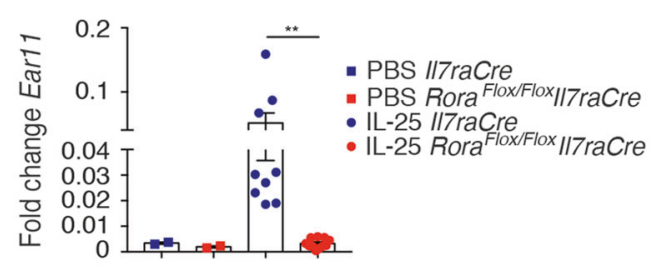

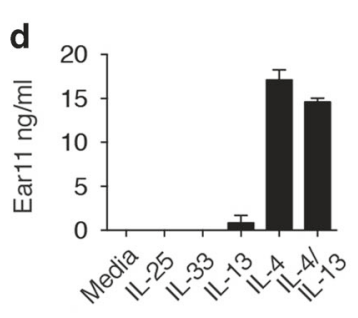

b

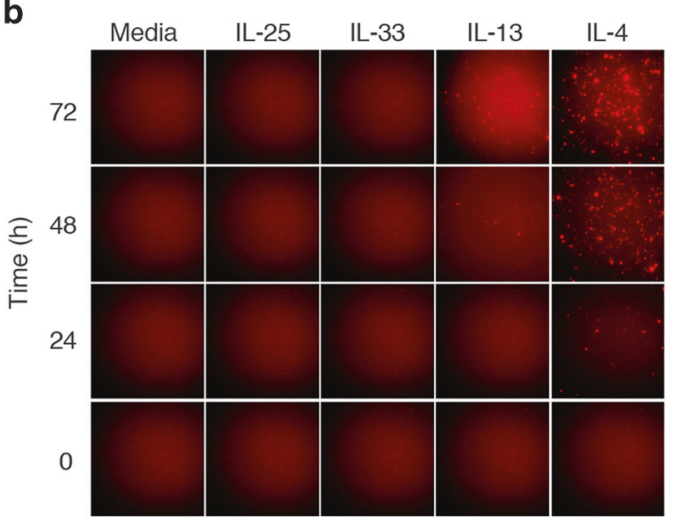

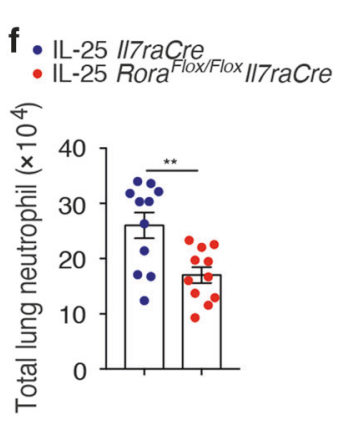

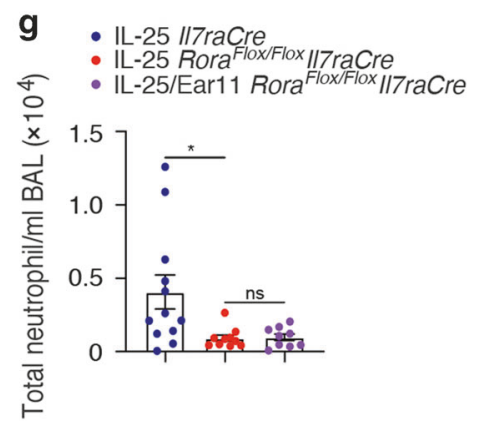

Fig. 4 Ear $11 \mathrm{mCherry}$ is expressed preferentially by alternatively activated macrophages. a Expression of Ear11, Retnla (Relma), and Arg1 by alveolar Ear11-negative or Ear11-positive macrophages sorted from Ear11 ${ }^{+/ C h}$ mice treated with intranasal IL-25. Assessed by qPCR. b Ear11 ${ }^{+/ C h}$ expression by peritoneal macrophages $\left(C D 45^{+} \mathrm{CD} 11 \mathrm{~b}^{+} \mathrm{F} 4 / 80^{+}\right)$cultured in a time-course in either media alone or media supplemented with rmlL-25, rmlL-33, rmlL-13, or rmlL-4 at $10 \mathrm{ng} / \mathrm{ml}$. Cells were imaged every $24 \mathrm{~h}$ using a Nikon HCA fluorescence microscope. c Ear 11 expression by peritoneal macrophages cultured as indicated (in b) for $72 \mathrm{~h}$, assessed by qPCR. Cells from 20 pooled mice, two wells/condition. $\mathbf{d}$ Ear1 1 protein concentration in cultures (as in b) assessed by ELISA. Data representative of two independent experiments. e Ear11 expression in sorted alveolar macrophages and $\mathbf{f}, \mathbf{g}$ total lung neutrophil numbers as assessed by flow cytometry, following two consecutive intranasal doses of IL-25 or IL-25 and Ear11. Data pooled from two independent experiments ( $n=4-6$ per experiment).

macrophages was not induced by culture with IL-5, IFN- $\gamma$, or TLR 1-9 agonists (data not shown).

Since the induction of Ear11 occurs within $24 \mathrm{~h}$ of IL-25 treatment, $^{34}$ we assessed whether IL-4 and IL-13-producing ILC2 were required for Ear11 expression and IL-25-induced neutrophil infiltration. ${ }^{5}$ ILC2-deficient (Rora ${ }^{f / f}$ IITraCre) and II7raCre control mice, ${ }^{8}$ were treated intranasally with two consecutive doses of IL-25 and $24 \mathrm{~h}$ later lung $\mathrm{CD} 45^{+} \mathrm{CD} 11 \mathrm{c}^{+} \mathrm{Si}-$ glecF ${ }^{+} \mathrm{F} 4 / 80^{+}$macrophages were sorted and tested for Ear11 expression by qPCR. Whilst IL-25 administration resulted in highly elevated levels of Ear11 in macrophages purified from II7raCre control mice, there was a near absence of Ear11 expression in macrophages derived from the ILC2-deficient mice (Fig. 4e). Furthermore, the absence of ILC2 also resulted in reduced neutrophil infiltration as compared with ILC2-sufficient controls upon IL-25 challenge, without affecting homoeostatic neutrophil numbers (Fig. 4f, Supplementary Fig. 6d). Interestingly, complementation of IL-25 challenge with rmEar11 was not sufficient to induce neutrophil recruitment in the lung of ILC2deficient mice (Fig. 4g).

These results demonstrate that the inducer cytokine IL-25 does not directly induce Ear11 in AAMs, but instead induces IL-4 and IL-13. This likely occurs via an ILC2-dependent mechanism. In turn, IL-4/IL-13-induced AAM upregulate Ear11 production and inducing IL-25-dependent lung neutrophilia.
Ear11 is required to maintain neutrophil homoeostasis in tissues Complementing the previous observations, we found that naive Ear11 $\mathrm{Ch} / \mathrm{Ch}$ mice had reduced numbers of neutrophils and macrophages in the lung, spleen, blood, and bone marrow in contrast to the increased neutrophilia observed in the Ear11Tg mice (Fig. $5 a-c)$. In line with neutrophil reduction, we observed decreased numbers of bone marrow promyelocytes and granulocyte-monocyte progenitors (as previously defined ${ }^{37}$ ), suggesting a potential role of Ear11 in neutrophil development (Supplementary Fig. 6e). CD4 T cells, CD8 T cells, B cells, ILC2, eosinophils, mast cells, basophils, common myeloid progenitors (CMPs) populations were all normal (data not shown).

Previous studies suggesting an association between Ear11 and lung allergy imply a potential role in type- 2 inflammation. ${ }^{38}$ Therefore, wild-type and Ear11-deficient mice were next treated intranasally with IL-25 for two consecutive days and $24 \mathrm{~h}$ after the last treatment tissues were analysed. In contrast to wild-type mice, Ear11 ${ }^{\mathrm{Ch} / \mathrm{Ch}}$ mice did not show increased neutrophil infiltration in the bronchoalveolar lavage (BAL) (Fig. 5d). In addition, wild-type mice and Ear11 Ch/Ch were treated with RWP extract for 5 consecutive days before analysis of the tissues $24 \mathrm{~h}$ after the last treatment. As expected, RWP treatment increased the numbers of macrophages in wild-type mice, and although the absence of Ear11 did not alter the numbers of macrophages significantly, we did observe fewer neutrophils in the lungs of the $\mathrm{Ear} 11^{\mathrm{Ch} / \mathrm{Ch}}$ mice 
a

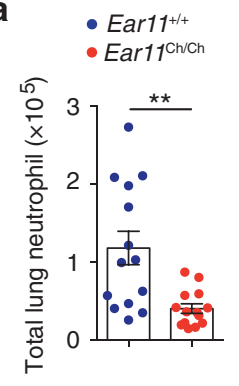

C

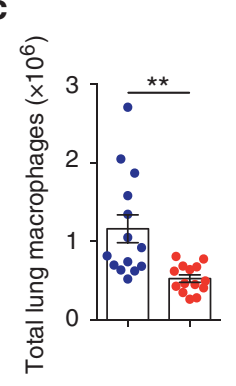

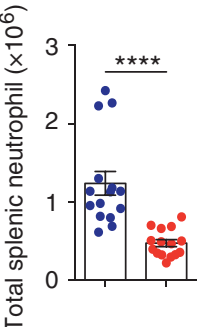

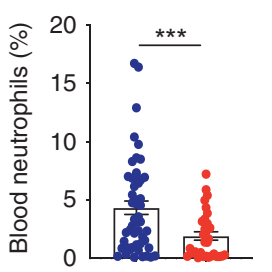

b

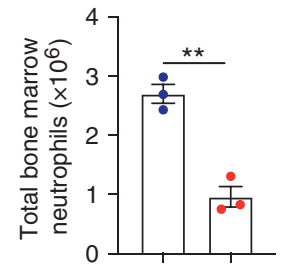

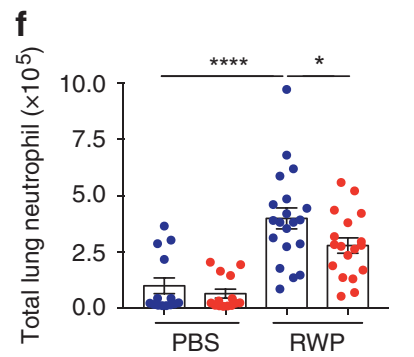
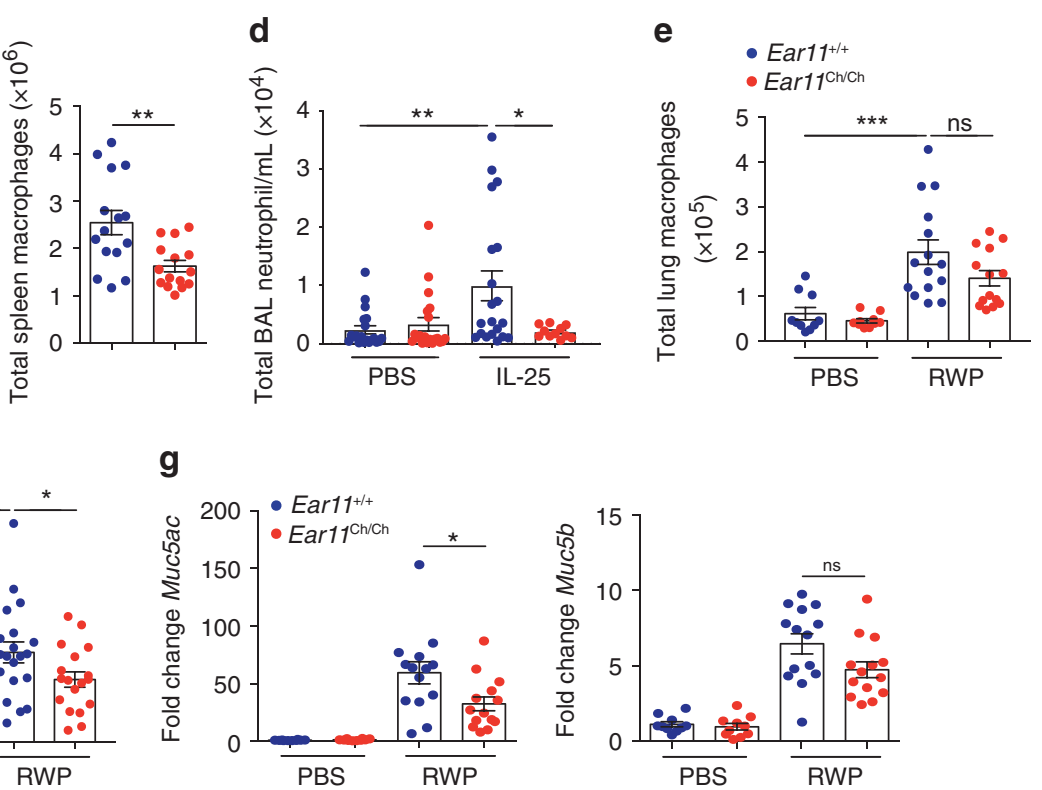

Fig. 5 Ear11 helps maintain both naive and type-2 dependent tissue neutrophilia. Flow cytometric analysis of total neutrophil numbers in a the lung and spleen, (data pooled from 3 independent experiments, $n=3-6$ per experiment), blood (data pooled from three independent experiments, $n=10-20$ per experiment), and $\mathbf{b}$ bone marrow (representative of two independent experiments, $n=6)$ of naive Ear1 $1^{+/+}$and Ear1 $1{ }^{C h / C h}$ mice. c Total number of lung and spleen macrophages in naive Ear $11^{+/+}$and Ear1 $1^{\mathrm{Ch} / \mathrm{Ch}}$ mice. $\mathbf{d}$ Total number of lung neutrophils in mice treated intranasally with either PBS or IL-25. e Total number of lung macrophages in mice treated intranasally with PBS or RWP. $\mathbf{f}$ Total number of lung neutrophils in mice treated intranasally with either PBS or RWP. g Quantitative PCR analysis of Muc5ac and Muc5b in lung tissue from e. $\mathbf{c}-\mathbf{g}$ Data representative of two independent experiments, $n=5-10$ per experiment.

(Fig. 5e, f). Recruitment of eosinophils to the lung in both IL-25 and RWP challenges was completely independent of Ear11 (data not shown). In addition, Muc5ac (but not Muc5b) expression in the lungs in response to RWP, assessed as an indicator of mucus production, was decreased in the absence of Ear11 (Fig. $5 \mathrm{~g}$ ).

In vitro assays have led to the proposal that human RNases have anti-helminthic properties. ${ }^{29}$ To investigate the potential in vivo role of Ear11 in gut-induced type-2 immunity to parasitic helminth infection, wild-type and Ear11 Ch/Ch mice were challenged with $\mathrm{N}$. brasiliensis larvae. Ear11-deficiency did not alter worm infectivity, cellular infiltration including neutrophils, or cytokine production as compared with wild-type controls in an acute infection model (Supplementary Fig. 7a-c). Furthermore, in a $\mathrm{N}$. brasiliensisinduced model of type-2 immune response resolution, in which lung tissues were analysed 32 days post infection, the numbers of macrophages and eosinophils were normal in Ear11-deficient mice. However, neutrophils were found to be reduced in number in Ear $11^{\text {Ch/Ch }}$ mice (Supplementary Fig. 7d).

We hypothesised that other members of the Ear gene family may be upregulated during type- 2 inflammation and be partially compensating functionally for the loss of Ear11 (e.g. normal worm clearance and recruitment to some extent of neutrophils). The RNase genes are located in two regions on mouse chromosome
14 (Supplementary Fig. 8a). We compared gene expression levels of known members of the Ear gene family (Ang, Ear1, Ear2, Ear5, Ear6, Ear10, and Ear14) in the lungs of wild-type and Ear11 $\mathrm{Ch} / \mathrm{Ch}$ mice (data not shown). Notably, Ang, a gene closely related to Ear11, was found to be more highly expressed in the lungs of Ear11 $\mathrm{Ch} / \mathrm{Ch}$ mice as compared with lung samples from wild-type mice (Supplementary Fig. 8b). These data demonstrate that the expression of Angiogenin increases in the absence of Ear11 raising the possibility that Ear11 and Ang genes may have redundant roles in inducing aspects of type- 2 immunity.

Together these results indicate that Ear11 contributes to the homoeostasis of neutrophil numbers in normal tissues and helps regulate neutrophil recruitment in response to type- 2 immunityinducing agents. However, despite being highly upregulated by IL25 , RWP and $N$. brasiliensis, in vivo, we were unable to demonstrate an essential role for Ear11 in type-2 inflammation.

Recombinant Ear11 provokes macrophage and neutrophil recruitment to the peritoneum

To determine the potential mechanism underlying the in vivo role of Ear11 in regulating neutrophils, a single dose of rmEar11 protein (Supplementary Fig. 4a, b), was administered intraperitoneally to mice before harvesting peritoneal lavage (PEL) cells 
a
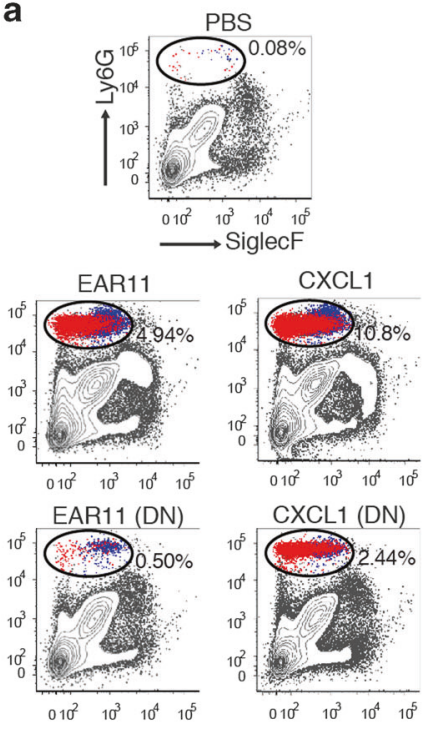

d
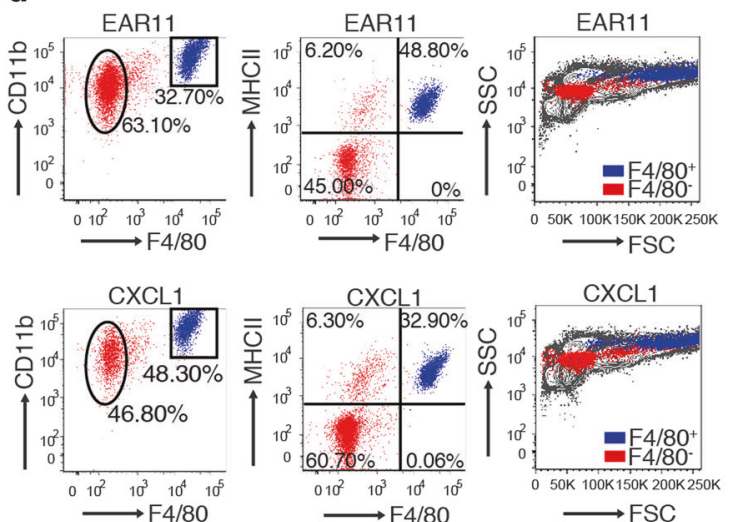

b

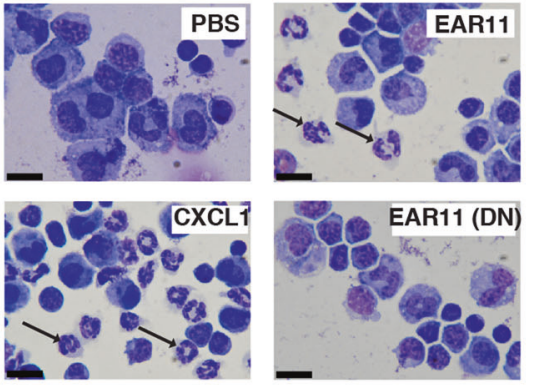

C

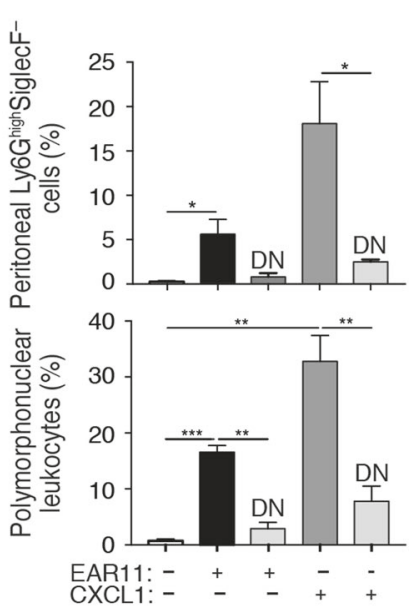

$\mathbf{f}$
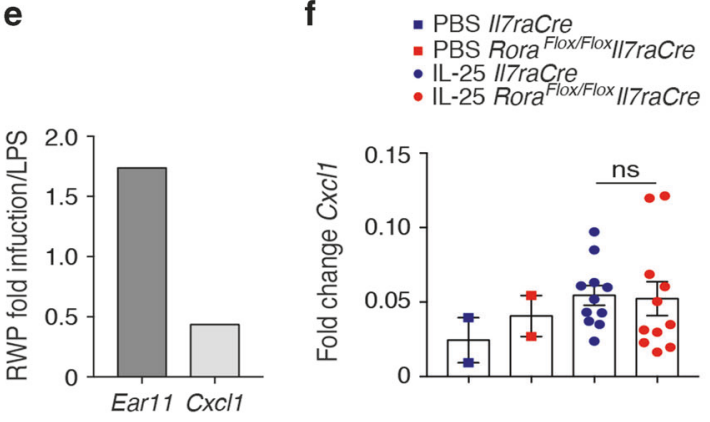

Fig. 6 Intraperitoneal administration of rmEar11 induces neutrophil and macrophage recruitment. a Flow cytometric analysis of peritoneal lavage cells from naive BALB/c mice treated (native or denatured (DN) protein) as shown (dot plots gated on LiveCD45 ${ }^{+}$cells), and b cytospin and Giemsa staining (representative images at $\times 100$ magnification, $20 \mu \mathrm{m}$ scale bar). c Quantification of Ly6G ${ }^{+}$SiglecF ${ }^{-}$cells from flow cytometry by fixed volume count and of polymorphonuclear leucocytes by differential count (cytospin, bottom panel). Data representative of three independent experiments $(n=3-4)$. d Phenotypic analysis of Ly6 $\mathrm{G}^{\text {high }}$ SiglecF ${ }^{-}$cells using CD11b, F4/80, MHCIl, and FSC vs. SSC. Cells identified by flow cytometry in the Ly $6 \mathrm{G}^{\text {high }}$ SiglecF ${ }^{-}$gate as 'macrophage' coloured blue and as 'neutrophil' coloured red. e Ratio of Ear11 and Cxcl1 gene expression from purified lung macrophages from wild-type mice challenged with ragweed pollen (RWP) or LPS. f Cxcl1 expression in sorted lung macrophages following two consecutive intranasal doses of IL-25. Data pooled from two independent experiments $(n=4-5)$.

$3 \mathrm{~h}$ later. This resulted in the rmEar11-dependent infiltration of $\mathrm{CD}_{4} 5^{+}$Ly6G $^{\text {high }}{ }^{\text {SiglecF }}{ }^{-}$cells that included neutrophils, as compared with PBS controls (Fig. 6a-c). Additional cell surface markers confirmed that rmEar11 recruited predominantly $\mathrm{Ly}_{6 \mathrm{G}}{ }^{\text {high }} \mathrm{Si}$ glecF ${ }^{-} \mathrm{CD} 11 \mathrm{~b}^{+} \mathrm{F} 4 / 80^{-} \mathrm{MHCl}^{-} \mathrm{SSC}^{\text {low }}$ neutrophils and to a lesser extent Ly6G ${ }^{\text {high }}$ SiglecF ${ }^{-} \mathrm{CD} 11 \mathrm{~b}^{+} \mathrm{F} 4 / 80^{+} \mathrm{MHCII}^{+} \mathrm{SSC}^{\text {high }}$ macrophages (Fig. 6d). Furthermore, histological analysis of purified Ear11-induced Ly $6 \mathrm{G}^{\text {high }}$ SiglecF ${ }^{-}$cells confirmed the infiltrate to be composed of $\sim 70 \%$ neutrophils and $\sim 30 \%$ macrophages (Fig. $6 \mathrm{~b}-\mathrm{d}$ and data not shown). The neutrophil-recruiting activity of rmEar11 was heat labile being lost when the rmEar11 protein was denatured, indicating that potential contamination with heat stable LPS was not responsible for the cellular infiltration (Fig. 6a-c). Interestingly, Ear11 was almost as potent at inducing neutrophil infiltration, as the neutrophil chemoattractant, CXCL1, that is a product characteristic of CAMs (Fig. 6a-d). Together, these results demonstrate that Ear11 provokes neutrophil, and to a lesser degree, macrophage recruitment, which appears to be similar in nature to the infiltrate induced following treatment with the CAM-associated CXCL1. However, Ear11 and CXCL1 were observed to be reciprocally expressed following challenge of mice with either LPS or RWP, with Ear11 characteristic of the type- 2 immune response initiated by RWP, and CXCL11 elicited by the type- 1 stimulant LPS (Fig. 6e), suggesting that they would each elicit their neutrophilic effect as part of different types of immunological response. Indeed, CXCL1 expression was unchanged in sorted macrophages taken from II7raCre control mice and ILC2-deficient mice treated intranasally with IL-25 (Fig. 6f).

Ear11 induces chemokinetic movement of neutrophils in the peritoneum

To directly assess the ability of Ear11 to induce chemotaxis or chemokinesis of cells, ${ }^{39}$ within the Ly $6 \mathrm{G}^{\text {high }}$ SiglecF ${ }^{-}$cell population, in vitro transwell migration assays were performed. To avoid cellular activation through Ly6G ligation during cell purification, 
a
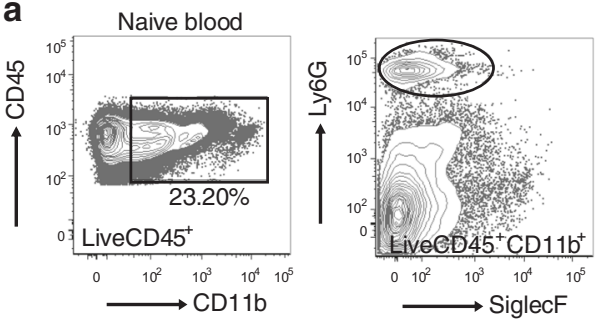

C
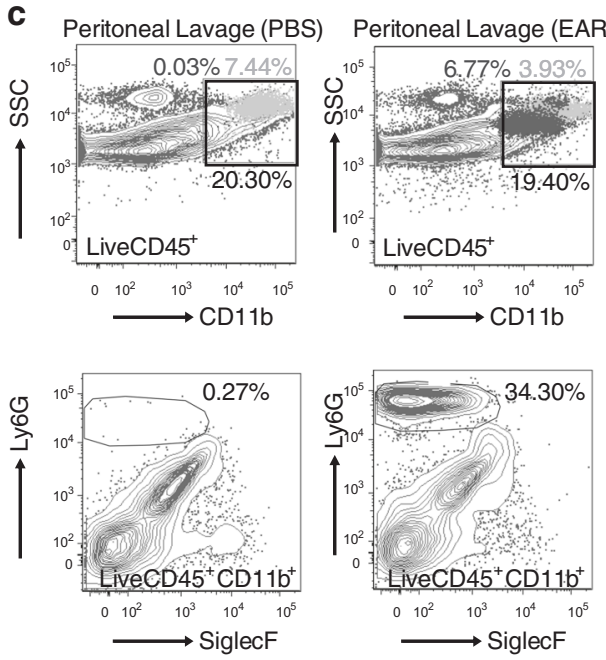

b

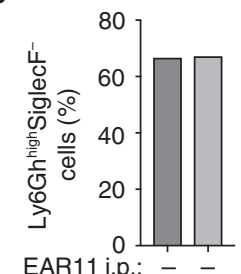

$-$
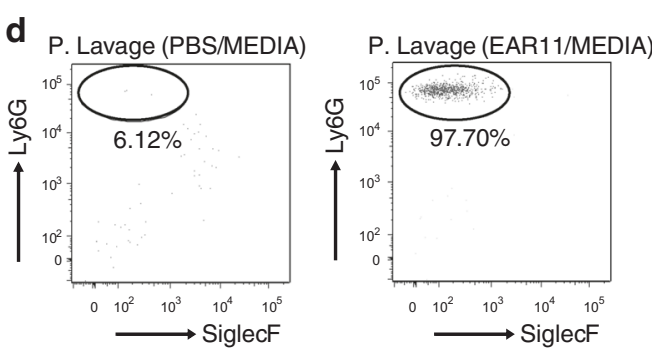

e

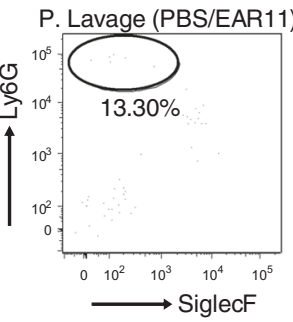

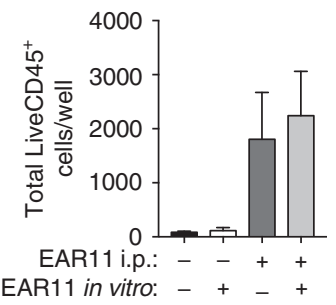

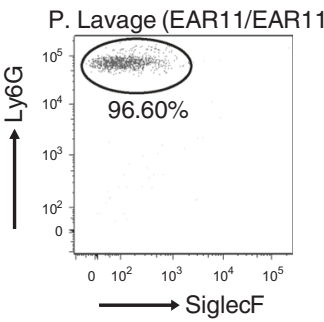

Fig. 7 Ear11 mobilises peritoneal neutrophils. a Naive LiveCD45 ${ }^{+} \mathrm{CD} 11 \mathrm{~b}^{+}$cells were sorted from blood, and stained with Ly6G and SiglecF to show the presence of neutrophils $\left(\mathrm{Ly} 6 \mathrm{G}^{\text {high }}\right.$ SiglecF ${ }^{-}$). $\mathbf{b}$ Sorted LiveCD $45^{+} \mathrm{CD} 11 \mathrm{~b}^{+}$cells were cultured in an in vitro transwell system with or without $20 \mathrm{ng} / \mathrm{ml}$ Ear11 and flow cytometry performed on cells which migrated through the membrane to show the percentage of $\mathrm{Ly}_{6 G^{\text {high }}}$ SiglecF ${ }^{-}$migratory neutrophils. c Peritoneal lavage cells sorted from PBS- or Ear11-treated mice as LiveCD45 ${ }^{+}$CD11 $b^{+}$. Top panel shows light blue cells CD $11 b^{+}$Ly6G $G^{\text {int }}$ and dark blue cells CD11 b ${ }^{+}$Ly6G ${ }^{\text {high }}$. Bottom panel shows neutrophils gated as Ly6 $G^{\text {high }}$ SiglecF ${ }^{-}$within LiveCD $45^{+} \mathrm{CD} 11 \mathrm{~b}^{+}$gate. d Sorted cells were cultured as in $\mathbf{b}$. e Total number LiveCD $45^{+}$migratory neutrophils collected following Ear11 treatment. Data are representative of two independent experiments.

the anti-Ly6G antibody staining was omitted and the total macrophage- and neutrophil-containing $\mathrm{CD}_{4} 5^{+} \mathrm{CD} 11 \mathrm{~b}^{+}$cell population was FACS purified from blood (Fig. 7a) and the migration of these previously unstimulated cells, through a transwell membrane, assessed over a 2-h period in response to Ear11. Although migration was detected this was not restricted to neutrophils, but included $\mathrm{CD} 45^{+} \mathrm{CD} 11 \mathrm{~b}^{+} \mathrm{Ly} 6 \mathrm{G}^{-}$cells, and was not augmented by the presence of Ear11 in the bottom well (Fig. 7b). These data suggest that Ear11 is not a chemotactic factor but is likely to be chemokinetic, i.e. it promotes the general non-directional migration of cells. ${ }^{39}$

To further address this possibility, we purified the total intraperitoneal macrophage- and neutrophil-containing $\mathrm{CD} 45^{+} \mathrm{CD} 11 \mathrm{~b}^{+}$cell population from mice $3 \mathrm{~h}$ after treatment of mice with either PBS or Ear11 (Fig. 7c). Cell migration through a transwell membrane over a 2 - $h$ period was determined in response to media alone or Ear11 in the bottom chamber. In the absence of exogenous Ear11 provocation of the mice, very few Ly6G $^{\text {high }}$ SiglecF ${ }^{-}$cells were present in the $\mathrm{CD}_{4} 5^{+} \mathrm{CD} 11 \mathrm{~b}^{+}$cell fraction purified from PBS-treated PEL. Consequently, the in vitro migration assays contained too few cells for meaningful analysis (Fig. 7d, e). However, pre-treatment of mice with Ear 11 resulted in the marked increase of $\mathrm{CD}_{4} 5^{+} \mathrm{CD} 11 \mathrm{~b}^{+} \mathrm{Ly}_{6 \mathrm{G}^{\text {high }}}$ SiglecF ${ }^{-}$cells in the $\mathrm{PEL}$, that were purified for assessment in migration assays. Interestingly, prior treatment of mice with Ear11 resulted in preferential migration of $\mathrm{CD}_{4} 5^{+} \mathrm{CD} 11 \mathrm{~b}^{+} \mathrm{Ly}_{6 \mathrm{G}^{\text {high }}}$ SiglecF ${ }^{-}$neutrophils through the membrane (Fig. 7d). In contrast, despite the presence of $\mathrm{CD}_{4} 5^{+} \mathrm{CD} 11 \mathrm{~b}^{+} \mathrm{Ly}_{6 \mathrm{G}^{-}}$cells in the $\mathrm{CD} 45^{+} \mathrm{CD} 11 \mathrm{~b}^{+}$cell population in the upper chamber these macrophages did not migrate under these conditions (data not shown). Notably, neutrophil migration was not increased with the inclusion of Ear11 in the lower chamber as compared with media alone controls (Fig. 7d, e), further suggesting that a 'chemokinetic migratory' neutrophil phenotype was induced in the neutrophils recruited to the peritoneum by in vivo treatment with Ear11.

Taken together the results demonstrate that Ear11 can provoke increased motility in neutrophils, suggesting that AAMs secreting Ear11 can act to mobilise neutrophils.

\section{DISCUSSION}

Type-2 immunity is characterised by a predominant eosinophilia and the appearance of AAMs, and whilst neutrophils are present they are relatively fewer in number. In part this is because AAMs, in contrast to CAMs, do not express chemoattractants such as CXCL1 that typically drive neutrophil recruitment. ${ }^{40,41}$ Our data suggest that Ear11 production predominantly from monocytes, AAMs, and alternatively activated DCs, contributes to maintaining neutrophil numbers in tissues during homoeostasis, and following type-2 immune activation. In the case of type- 2 responses this is at least partially dependent on ILC2. Thus, in an IL-25 driven type- 2 immune microenvironment an alternate Ear11-mediated mechanism has arisen to ensure that neutrophils can contribute to the immune reaction without the requirement for $\mathrm{TLR}$ and IFN- $\gamma$ stimulated CAM-derived neutrophil chemoattractants and the escalation of an unwanted and counter-productive pro-inflammatory response. 
In the mouse, a large family of small ribonucleases (Ear) show notable sequence and structural homology to human EDN and ECP. ${ }^{42-44}$ The secreted Ear proteins are characterised by an Nterminal signal peptide followed by a ribonuclease $A$ domain containing conserved catalytic histidines and a lysine. ${ }^{45}$ Mouse Ear11 (also known as Rnase2a) transcripts are expressed at basal levels in naive tissues, but can be highly upregulated in response to type- 2 immune activation. ${ }^{35}$ Indeed, Ear11 expression has been shown to be elicited in lung alveolar macrophages following the induction of ovalbumin-induced airways inflammation, ${ }^{38}$ and in response to short and long-term mouse models of allergic asthma. ${ }^{46,47}$ Similarly, administration of the type- 2 initiator cytokines IL-25 or IL-33 also induced Ear11 gene expression. ${ }^{34,35}$ Although most members of this RNase A gene family have not been studied extensively, a recent report indicated that Ear11 may function in vitro as a chemoattractant for tissue macrophages independently of TLR2 signalling. ${ }^{35}$

Although macrophages represent a spectrum of phenotypes influenced by local environmental cues and stimuli, distinct polarised subsets have been described. ${ }^{10}$ CAMs arise in response to stimulation via IFN- $\gamma$ and TLR ligation leading to their production of inflammatory cytokines, nitric oxide, and chemokines such as CXCL1 that recruit neutrophils to sites of microbial infection. By contrast, the type-2 cytokines IL-4 and IL-13 support AAMs that are often considered anti-inflammatory, promoting quiescence and repair, and restricting inflammatory responses characterised by CAMs. This response helps to restore the damage resulting from parasitic worm invasion, but may lead to inappropriate tissue repair and fibrosis in allergic disease. Macrophages exhibiting an AAM phenotype (also known as M2 or M(IL-4)), characterised by the expression of high levels of Relma and $\operatorname{Arg} 1$, have been reported in multiple tissues, ${ }^{48}$ suggesting a broad physiological role, possibly in maintaining immune homoeostasis and preventing unwanted inflammation. Indeed, tissueresident ILC2s have been shown to produce IL-4 and IL-13 that sustain AAMs for the establishment of inflammatory thresholds in neonate and adult lungs, ${ }^{49}$ and in adipose tissue to protect from obesity. ${ }^{50}$ Such an environment may be maintained through tissue-resident macrophages self-renewing and proliferating in response to IL-4 without additional recruitment. ${ }^{51}$

Using a new Ear11 $\mathrm{Ch/+}$ knock-in reporter mouse, we demonstrated Ear11 expression in vivo across multiple tissues, cell types, and models of immune activation. Ear11 was highly restricted to monocytes $\left(\mathrm{CD} 11 \mathrm{~b}^{+} \mathrm{Ly}^{+} \mathrm{C}^{+} \mathrm{CD} 115^{+}\right)$, dendritic cells $\left(\mathrm{CD} 11 \mathrm{c}^{+} \mathrm{MHClI}^{\text {high }}\right)$, and macrophages $\left(\mathrm{CD} 11 \mathrm{~b}^{+} \mathrm{F} 4 / 80^{+}\right)$and was only induced by type- 2 cytokines, allergic challenges, and parasitic infection, but not by pneumoviral (PVM) infection or bacterial (LPS) provocation. Although Ear11 expression was induced by IL25 and IL-33 in vivo, these cytokines did not upregulate Ear11 production directly from macrophages, but instead this required IL-4, in line with a report that IL-4 provoked Ear11 transcripts from alveolar macrophages. ${ }^{35}$ AAMs are induced in an IL-4Ra1- and STAT6-dependent manner and are characterised by expression of molecules including the mannose receptor, Ym1, Arg1, Relma, and Fizz1. ${ }^{52-54}$ Our in vivo analysis has established that Ear11 expression was limited to Relma-positive AAMs. This discovery prompts the future investigation of the syntenic human molecules, EDN and ECP, which may have as yet unappreciated roles in AAM biology and indeed these molecules do seem to be expressed at low levels in RNASeq datasets taken from human AAM cultures. ${ }^{55}$

Exploiting several complementary approaches, including Ear11 overexpression in transgenic mice, Ear11 ablation and exogenous Ear11 administration, we demonstrate here that Ear11 is a monocyte- and AAM-secreted regulator of neutrophil homoeostasis and migration. Consequently, Ear11-enhanced neutrophil recruitment to tissues, such as lung and spleen, was compromised in Ear11-deficient mice. Conversely, mice overexpressing Ear11, had increased neutrophil infiltration to peripheral tissues, but not in bone marrow, perhaps reflecting the normal constitutive naive expression of Ear11 here. In addition, when rmEar11 protein was given to mice it provoked a predominantly neutrophilic cell infiltration, and to a lesser extent macrophage recruitment, within $3 \mathrm{~h}$ of its administration. However, administration of Ear11 to ILC2-deficient mice was not sufficient to promote recruitment of neutrophils, suggesting that further, yet to be defined factors are also necessary. Taken together, these results demonstrate that Ear11 production by AAMs represents a novel mechanism for neutrophil maintenance and recruitment to sites of type-2 immunity. Though the role of neutrophils in type-2 immune responses remains controversial, it has been shown that an early migration of neutrophils to the site of infection is necessary to control worm burden. ${ }^{19,56,57}$ Our data using alarmin- and allergeninduced type-2 models support these previous observations. However, other studies suggest production of Th2-derived IL-4 and IL-13 later in the type-2 response can also inhibit neutrophilia. ${ }^{58-60}$ Our data suggest that early IL-4 production, from cell types such as ILC2s, may be required for timely neutrophil infiltration. Thus, these apparently contradictory observations may be reconciled by considering a differential involvement of neutrophils in different stages of the type- 2 response. ${ }^{61}$

Interestingly, in naive Ear11-deficient mice there were also fewer neutrophils in the tissues and fewer neutrophil progenitors in the bone marrow, suggesting that in the steady state Ear11 helps to maintain homoeostatic numbers of neutrophils. Further studies are required to determine whether this results from effects on development, proliferation, or survival (or a combination of the three). Ear11 may therefore poise neutrophil infiltration in a manner similar to the way that IL-5 and eotaxin regulate eosinophils. $^{62-64}$ The precise pathways required to maintain neutrophils at tissue sites during homoeostasis are not well characterised, but the rate of neutrophil production, storage in and egress from the bone marrow, and survival in, and movement out of the blood are all proposed to play a role in this 'neutrostat'. ${ }^{65}$ Our results suggest that Ear11 production by monocytes and AAMs contributes to this process. Interestingly, Ear11 is part of a family of RNases and we noted that in the absence of Ear11 the highly related molecules Angiogenin was upregulated, suggesting that compensatory mechanisms may arise between members of this family. Thus, it may be necessary to remove two or more of these genes to fully explore the impact of RNase-regulated neutrophil induction on immune function.

The rapid influx of neutrophils following intraperitoneal injection of rmEar11 clearly demonstrated its capacity to stimulate neutrophil, and to a lesser extent macrophage, recruitment to the peritoneal cavity. Notably, the migratory effect of Ear11 was only marginally less potent than exogenously administered CXCL1. Interestingly, when we harvested cells from the peritoneal lavage of Ear11-provoked mice we found that the neutrophil component was highly migratory in vitro, whilst these assays were unable to detect macrophages migrating in the same time-frame. It is also noteworthy that once activated in vivo the neutrophils did not respond chemotactically to additional Ear11 provided in the assay system, implying that they are responding chemokinetically to Ear11. These assays could only be performed using cells from Ear11-stimulated mice since these populations were absent from the peritoneal lavage in the absence of Ear11 provocation. In vitro studies carried out previously suggested that rmEar 11 provided a chemoattractant signal to naive $\mathrm{F} 4 / 80^{+} \mathrm{CD} 11 \mathrm{c}^{-}$macrophages within the total splenocyte population assayed, with little effect on Ly6G ${ }^{+}$cells. ${ }^{35}$ However, we now demonstrate that in vivo the main cell target of Ear11-induced migration is neutrophils, with a lesser but still significant contribution to macrophage recruitment. This difference between the two studies may result from the tissue source of the cells under study (e.g. spleen versus peritoneal lavage) and the importance of maintaining tissue and cellular environments using in vivo approaches. 
In conclusion, we have demonstrated a novel in vivo role for Ear11, a close relative of human ECP and EDN proteins, in neutrophil recruitment and maintenance in tissues. Consequently, Ear11 produced by IL-4-activated AAMs as part of type-2 immune reactions, is exquisitely placed to support the infiltration of neutrophils in response to immune challenges that fail to elicit CXCL1-producing pro-inflammatory CAM (Supplementary Fig. 9). Our data also raise the possibility that human RNase molecules may have similar roles in humans and contribute to neutrophilic asthma.

\section{METHODS}

Mice

Overexpression of Ear11 (Rnase2a) in all tissues was achieved by microinjection of wild-type Ear11 CDNA, under the transcriptional control of CAG promoter in the pCAGGS vector (Supplementary Table 1), into F1 fertilised eggs. Two founder Ear11 transgenic (Tg) lines were identified. Ear11Tg mice did not breed and in vitro fertilisation was used to maintain two of the founder lines (mouse identifier 64572 and 71970).

Ear $11 \mathrm{mCherry}$ reporter mice $\left(\right.$ Ear $\left.11^{\mathrm{Ch} / \mathrm{Ch}}\right)$ mice were generated by replacing exon 2 of Ear 11 with the mCherry fluorescent protein coding sequence followed by a floxed neomycin resistance gene in JM8.6 (C57BL/6) embryonic stem cells by homologous recombination. Embryonic stem cell clones in which a successful targeting event had occurred were identified using $5^{\prime}$ and $3^{\prime}$ probes (Supplementary Table 1) by Southern blot analysis and used to generate Ear11 $1^{+/ C h}$ mice. The neomycin gene was removed in the germline by interbreeding $\mathrm{Ear} 11^{\mathrm{Ch} / \mathrm{Ch}}$ with a Crerecombinase expressing strain. The line was then also backcrossed onto the $\mathrm{BALB} / \mathrm{c}$ background for six generations.

For bone marrow transfer experiments B6SJL (CD45.1) mice were sub-lethally irradiated with two doses of $4.5 \mathrm{~Gy}$ Y-irradiation. On the same day they received $1-2 \times 10^{6}$ bone marrow cells (fresh or frozen stocks), from either C57BL/6 (CD45.2) or Ear11Tg (CD45.2) mice, by intravenous injection. Mice were reconstituted for at least 6 weeks.

II7raCre and Rora ${ }^{f / f} / 17 \mathrm{raCre}$ mice are previously described. ${ }^{8}$ Ageand sex-matched mice at 6 weeks old or above were used in experiments. Mice were housed in specific pathogen-free conditions. All experiments were undertaken with the approval of the UK Home Office.

Generation of rmEar11 protein and anti-mouse Ear11 antibodies Recombinant mouse (rm)Ear11 was expressed as a $6 x \mathrm{His}$ fusion protein in Sf9 cells using Bac-to-Bac Baculovirus Expression System (Thermo Fisher Scientific) and purified from cell culture supernatants on a Nickel NTA resin column (GE Healthcare) followed by gel filtration on HiLoad 16/60 Superdex 75 column (GE Healthcare) and then HisTrap HP column (GE Healthcare). Purified protein was dialysed into sterile, endotoxin-free PBS. Endotoxin concentration was quantified at $32 \mathrm{EU} / \mathrm{mg}$ using the Limulus/Amebocyte Lysate (LAL) assay according to manufacturer's instructions (Pierce, ThermoFisher).

A male rat was immunised by sub-cutaneous injection of $100 \mu \mathrm{g}$ of rmEar11 combined in a 1:1 ratio with TiterMax (Sigma-Aldrich) on day 0,28 , and 32 . A single intravenous injection of $50 \mu \mathrm{g}$ of Ear11 was given on day 84. Monoclonal antibodies were generated by standard protocols.

Ear11 protein detection

Ear11 levels in supernatants were assessed using a Meso Scale Diagnostics (MSD) assay. MSD standard binding plates (Meso Scale Diagnostics) were coated with polyclonal anti-Ear11 serum (1:2500 dilution), blocked with 5\% Blocker A (Meso Scale Diagnostics) and incubated with standards (serial dilution of rmEar11) and/or samples overnight. Detection antibody, biotinylated monoclonal rat antimouse Ear11 antibody (clone BC2.12), was added at $2 \mu \mathrm{g} / \mathrm{ml}$, followed by streptavidin SULFO-TAG (Meso Scale Diagnostics) and 2x Read buffer T (Meso Scale Diagnostics). The electrochemiluminescent signal was read on a Sector S 600 plate reader.

\section{Mouse challenge protocols}

In all instances recombinant proteins, extracts, and LPS were given in endotoxin-free PBS (Sigma-Aldrich) or PBS as a control.

rmlL-25 ( $2 \mu \mathrm{g} /$ dose, made in-house, Janssen Pharmaceuticals), rmlL-33 ( $0.5 \mu \mathrm{g} /$ dose, Peprotech) or rmEar11 ( $2 \mu \mathrm{g} /$ dose $)$ were given on 2 or 3 consecutive days (as indicated) and tissues harvested $24 \mathrm{~h}$ after the final dose. rmEar11 ( $2 \mu \mathrm{g} /$ dose) or CXCL1 $(0.5 \mu \mathrm{g} / \mathrm{ml}, \mathrm{R}$ and D Systems; $<100 \mathrm{EU} / \mathrm{mg}$ protein) were given as a single dose and tissues were harvested at $12 \mathrm{~h}$ post injection. rmEar11 and CXCL1 were boiled for $60 \mathrm{~min}$ to denature the protein.

RWP (100-200 $\mu \mathrm{g} /$ dose, RWP, Ambrosia artemisiifolia, short form, Greer Laboratories) was given intranasally on 5 consecutive days. All tissues were harvested $24 \mathrm{~h}$ after the final dose. Alternatively, three doses RWP were administered intranasally over 3 days and lung $\mathrm{CD}_{4} 5^{+} \mathrm{CD} 11 \mathrm{c}^{+} \mathrm{F} 4 / 80^{+}$SiglecF $^{+}$alveolar macrophages purified $24 \mathrm{~h}$ after the final dose. Macrophage gene expression was analysed by $\mathrm{qPCR}$.

LPS (1-2 $\mu \mathrm{g} /$ dose, Ultrapure LPS-EB from Escherichia coli 0111: B4, InvivoGen) was given intranasally on three consecutive days. Analyses were performed $24 \mathrm{~h}$ after the final dose. In some experiments, lung $\mathrm{CD}_{4} 5^{+} \mathrm{CD} 11 \mathrm{c}^{+} \mathrm{F} 4 / 80^{+}$SiglecF $^{+}$alveolar macrophages were purified and gene expression analysed by qPCR.

Mouse Infection models

Mice were inoculated sub-cutaneously with 500 viable third-stage $N$. brasiliensis larvae in endotoxin-free PBS and tissues were harvested on day $4,7,10$, and 32 post infection.

A single intranasal dose of 50 PFU of PVM (strain J3666, gift from Prof. Easton) was given in PBS or PBS alone. Tissues were analysed at day 2,5 , and 12 post infection.

Cell preparation and flow cytometry

Single lung cell suspensions were prepared by incubating finely chopped tissue with $720 \mu \mathrm{g} / \mathrm{ml}$ collagenase D (Roche) in RPMI (Invitrogen) at $37^{\circ} \mathrm{C}$ for $30 \mathrm{~min}$, passing the tissue through a $70 \mu \mathrm{m}$ cell strainer, centrifugation through $30 \%$ Percoll (GE Healthcare) in RPMI and lysing RBCs. Single spleen, mediastinal, and mesenteric lymph node cell suspensions were prepared by passing the tissue through a $70 \mu \mathrm{M}$ cell strainer (except when preparing cells for macrophage subpopulation analysis where tissue was treated with collagenase D, as for lung) and lysing RBCs. Single bone marrow and peritoneal cell suspensions were prepared by flushing the femur and tibia, or peritoneum with endotoxin-free PBS and lysing RBCs. Peripheral blood leukocytes were prepared using Whole Blood Lysing Reagent Kit (Beckman Coulter) according to the manufacturer's instructions.

Single cell suspensions were incubated with anti-CD16/CD32 antibody (Fc block, clone 93) followed by fluorochrome-conjugated antibodies (Biolegend, unless otherwise indicated) against: mouse Arginase 1 (A1exF5), B220 (clone RA3-6B2), CD3 (clone 145-2C11), CD4 (clone H129.19, BD Biosciences), CD8 (clone 53-6.7), CD11b (clone M1/70), CD11c (clone N418), CD19 (clone eBio 1D3), CD45 (clone 30-F11), CD45.1 (clone A20), CD45.2 (clone 104), CD115 (clone AFS98), CD127 (clone SB/199), F4/80 (clone BM8), FceR1 (clone MAR1), Gr-1 (RB6-8C5), ICOS (clone 398.4A), Ly6C (clone HK1.4), Ly6G (clone 1A8-Ly6g), MHCII (M5/114.15.2, Biolegend), Relma (DS8RELM, eBioscience), SiglecF (E50-2440, BD Biosciences). All samples were co-stained with a cell viability dye (Fixable dye eFluor780, Invitrogen). For analysis samples were run on an LSR Fortessa system (BD Biosciences). For cell sorting an iCyt Synergy (70 $\mu \mathrm{m}$ nozzle, Sony Biotechnology) was used.

Unless otherwise stated alveolar macrophages were defined as $\mathrm{CD}_{4} 5^{+} \mathrm{CD} 11 \mathrm{C}^{+} \mathrm{F} 4 / 80^{+}$SiglecF ${ }^{+}$, eosinophils as $\mathrm{CD}^{+} 5^{+} \mathrm{CD} 11 \mathrm{c}^{-} \mathrm{F} 4 /$

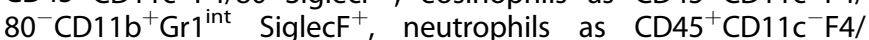




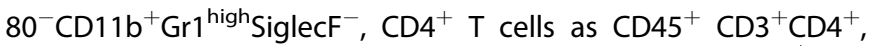
and innate lymphoid type-2 cells (ILC2s) as $\mathrm{CD}_{4} 5^{+} \mathrm{Lin}^{-}$ (CD3CD4CD8CD19 CD11bCD11cFceR1) CD127 ${ }^{+}$ICOS $^{+}$.

Immunofluorescence imaging

Lung and FALC were prepared by fixation in $4 \%$ paraformaldehyde at $4{ }^{\circ} \mathrm{C}$ for $1 \mathrm{~h}$, incubating in $20 \%$ sucrose overnight for cryoprotection, and embedding in $15 \%$ sucrose $/ 7.5 \%$ gelatin diluted in PBS. Overall, $12 \mu \mathrm{m}$ frozen sections were cut using a Leica CM $3050 \mathrm{~S}$ cryostat. Sections were pre-blocked/permeabilized in PBS containing 3\% BSA and $0.05 \%$ Triton X-100 and stained with primary antibodies anti-mCherry (M11217, Invitrogen), anti-Relma (500-P214, PeproTech), or anti-actin a-smooth muscle FITC conjugated (1A4, Sigma), followed by secondary antibodies anti-rat IgG Alexa Fluor 568 (A11077, Life Technologies) and anti-rabbit IgG Alexa Fluor 488 (A21206, Life Technologies) for $1 \mathrm{~h}$ at the room temperature, or directly conjugated CD3 (clone 145-2C11), B220 (clone RA3-6B2), and CD169 (clone 3D6.112). Image acquisition was performed using either a Zeiss LSM 780 confocal microscope and ZEN 2010 acquisition software or a Nikon HCA fluorescence microscope and NIS-Elements 4.30.01 acquisition software for live cell imaging. Adobe Photoshop 11.0.2 was used to adjust brightness, contrast and colour balance (changes were applied to all images in equal measure).

In vitro culture of primary cells

Sorted peritoneal macrophages were cultured for $72 \mathrm{~h}$ in RPMI media (Gibco) with 10\% fetal bovine serum (Labtech), 1\% penicillin/streptomycin and $100 \mu \mathrm{M}$ 2- $\beta$-mercaptoethanol (BDH Biochemicals) with or without $10 \mathrm{ng} / \mathrm{ml} \mathrm{IL-33} \mathrm{(Biolegend),} 10 \mathrm{ng} /$ $\mathrm{ml} \mathrm{IL-25} \mathrm{(Janssen),} 10 \mathrm{ng} / \mathrm{ml} \mathrm{IL-13} \mathrm{(eBioscience),} \mathrm{or} 10 \mathrm{ng} / \mathrm{ml} \mathrm{IL-4}$ (R\&D Systems). Cell free supernatants were collected for ELISA and cells were lysed using Trizol LS reagent (Life Technologies) for RNA extraction.

Cells were flushed from the bone marrow through a $70 \mu \mathrm{m}$ cell strainer and incubated with the following fluorochrome-conjugated antibodies against: CD5 (clone 53-7.3), CD117 (clone 2B8), Sca1 (clone D7), CD34 (clone RAM34), CD16/32 (clone 93), as well as lineage markers as previously described (CD11b, B220, Gr1, TER119, CD19). Fixable viability dye efluor780 (Invitrogen) was also added. CMP cells were then sorted as LiveLineage ${ }^{-} \mathrm{CD} 5^{-} \mathrm{CD} 117^{+} \mathrm{S}-$ $\mathrm{ca}^{-} \mathrm{CD} 16 / 32^{\text {int }} \mathrm{CD} 34^{+}$and plated at 100 cells/well in MethoCult ${ }^{\mathrm{TM}}$ with GF M3434 media (STEMCELL Technologies). Colony number and type were analysed at day 9 using the STEMvision system with the custom mouse CSC algorithm.

Live $\mathrm{CD} 45^{+} \mathrm{CD} 11 \mathrm{~b}^{+}$cells were sorted from peritoneal lavage of PBS- and Ear11-treated mice, or from naive blood and plated on 24 well transwell inserts (PET, $3.0 \mu \mathrm{m}$ pore, Falcon) at between 2.5 and $3.0 \times 10^{5}$ cells/well. Media alone or $10 \mathrm{ng} / \mathrm{ml} \mathrm{rmEar11}$ was added to the bottom well and cells were incubated at $37^{\circ} \mathrm{C}$ for 2 $\mathrm{h}$, after which cells that had migrated through the membrane were subject to flow cytometry.

Reverse-transcription (RT)-PCR and real-time quantitative (q) PCR RNA was isolated using RNA Bee reagent (Amsbio) or Trizol LS reagent (Life Technologies) according to the manufacturer's instructions. All RNA samples were treated with DNase I using RNeasy Micro Kit (Qiagen) and reverse-transcribed into complementary DNA using Super-RT enzyme (HT Biotechnology). RT-PCR was performed using KOD Hot Start DNA polymerase (Novagen) and self-designed primers (Table S1). Products were run on a $2 \%$ agarose gel and visualised using a Bio-Rad ChemiDoc XRS + System and Image Lab 5 software. Real-time qPCR was performed using SYBR Green mastermix (Applied Biosystems) and selfdesigned primers or Taqman Universal PCR master mix (Applied Biosystems) and commercially available Taqman gene expression assays (Table S1, Applied Biosystems). Samples were run on the ViiA7 real-time PCR system (Applied Biosystems).
Histology

Tissue was fixed in 10\% formalin overnight and paraffin-embedded. Sections were stained with haematoxylin and eosin. Image acquisition was performed using a Nikon Labophot-2 biological microscope and Camera Control Pro 2 software. Peritoneal lavage cells were subject to cytospin and differential counting.

Statistical analyses

Results are shown as mean \pm SEM. Results were considered significant at ${ }^{*} P \leq 0.05 ;{ }^{* *} P \leq 0.01 ;{ }^{* * *} P \leq 0.001 ;{ }^{* * * *} P \leq 0.0001$. Statistical analyses were performed using Prism version 6.0 .

\section{ACKNOWLEDGEMENTS}

We are grateful to the Ares staff, genotyping facility, and flow cytometry core for their technical assistance. We thank Olga Perisic for help with Ear11 protein production. The ES cell line JM8.6 was provided by the Wellcome Sanger Institute.

\section{AUTHOR CONTRIBUTIONS}

V.P. designed and performed experiments and wrote the paper. M.G., N.R-R., M.S., H.E.J., M.W.D.H., J.A.W., B.M.J.R., L.F.D., M.H., R.P., G.K., M.W., A.J.E., C.A.O., D.G.K., P.F.G., J.L.B. performed experiments, provided advice on experimental design and interpretation, and commented on the manuscript. J.L.B. and A.N.J.M. supervised the project, designed the experiments and wrote the paper.

\section{FUNDING}

This study was supported by grants from the UK Medical Research Council (U105178805), Wellcome Trust (100963/Z/13/Z).

\section{ADDITIONAL INFORMATION}

The online version of this article (https://doi.org/10.1038/s41385-020-0298-2) contains supplementary material, which is available to authorized users.

Competing interests: A.N.J.M. has grant funding from GSK and AZ/Medlmmune.

Publisher's note Springer Nature remains neutral with regard to jurisdictional claims in published maps and institutional affiliations.

\section{REFERENCES}

1. Hardman, C. S., Panova, V. \& McKenzie, A. N. IL-33 citrine reporter mice reveal the temporal and spatial expression of IL-33 during allergic lung inflammation. Eur. J. Immunol. 43, 488-498 (2013).

2. Cheng, D. et al. Epithelial interleukin-25 is a key mediator in Th2-high, corticosteroid-responsive asthma. Am. J. respiratory Crit. Care Med. 190, 639-648 (2014).

3. Gerbe, F. et al. Intestinal epithelial tuft cells initiate type 2 mucosal immunity to helminth parasites. Nature 529, 226-230 (2016).

4. von Moltke, J., Ji, M., Liang, H. E. \& Locksley, R. M. Tuft-cell-derived IL-25 regulates an intestinal ILC2-epithelial response circuit. Nature 529, 221-225 (2016).

5. Neill, D. R. et al. Nuocytes represent a new innate effector leukocyte that mediates type-2 immunity. Nature 464, 1367-1370 (2010).

6. Moro, K. et al. Innate production of $\mathrm{T}(\mathrm{H}) 2$ cytokines by adipose tissue-associated c-Kit(+)Sca-1(+) lymphoid cells. Nature 463, 540-544 (2010).

7. Price, A. E. et al. Systemically dispersed innate IL-13-expressing cells in type 2 immunity. Proc. Natl Acad. Sci. USA 107, 11489-11494 (2010).

8. Oliphant, C. J. et al. MHCll-mediated dialog between group 2 innate lymphoid cells and CD4(+) T cells potentiates type 2 immunity and promotes parasitic helminth expulsion. Immunity 41, 283-295 (2014).

9. Halim, T. Y. et al. Group 2 innate lymphoid cells license dendritic cells to potentiate memory TH2 cell responses. Nat. Immunol. 17, 57-64 (2016).

10. Gordon, S. \& Martinez, F. O. Alternative activation of macrophages: mechanism and functions. Immunity 32, 593-604 (2010).

11. Wilson, M. S. \& Wynn, T. A. Pulmonary fibrosis: pathogenesis, etiology and regulation. Mucosal Immunol. 2, 103-121 (2009).

12. Pesce, J. T. et al. Retnla (relmalpha/fizz1) suppresses helminth-induced Th2-type immunity. PLoS Pathog. 5, e1000393 (2009).

13. Pesce, J. T. et al. Arginase-1-expressing macrophages suppress Th2 cytokinedriven inflammation and fibrosis. PLoS Pathog. 5, e1000371 (2009). 
14. Rodriguez-Sosa, M. et al. Chronic helminth infection induces alternatively activated macrophages expressing high levels of CCR5 with low interleukin-12 production and Th2-biasing ability. Infect. Immun. 70, 3656-3664 (2002).

15. Varin, A., Mukhopadhyay, S., Herbein, G. \& Gordon, S. Alternative activation of macrophages by IL-4 impairs phagocytosis of pathogens but potentiates microbial-induced signalling and cytokine secretion. Blood 115, 353-362 (2010).

16. Allen, J. E., Sutherland, T. E. \& Ruckerl, D. IL-17 and neutrophils: unexpected players in the type 2 immune response. Curr. Opin. Immunol. 34, 99-106 (2015).

17. Bonne-Annee, S. et al. Human and mouse macrophages collaborate with neutrophils to kill larval Strongyloides stercoralis. Infect. Immun. 81, 3346-3355 (2013).

18. Chen, F. et al. Neutrophils prime a long-lived effector macrophage phenotype that mediates accelerated helminth expulsion. Nat. Immunol. 15, 938-946 (2014).

19. Sutherland, T. E. et al. Chitinase-like proteins promote IL-17-mediated neutrophilia in a tradeoff between nematode killing and host damage. Nat. Immunol. 15, 1116-1125 (2014).

20. Pesce, J. T. et al. Neutrophils clear bacteria associated with parasitic nematodes augmenting the development of an effective Th2-type response. J. Immunol. 180, 464-474 (2008).

21. Al-Qaoud, K. M. et al. A new mechanism for IL-5-dependent helminth control: neutrophil accumulation and neutrophil-mediated worm encapsulation in murine filariasis are abolished in the absence of IL-5. Int Immunol. 12, 899-908 (2000).

22. Malik, A. \& Batra, J. K. Antimicrobial activity of human eosinophil granule proteins: involvement in host defence against pathogens. Crit. Rev. Microbiol 38, 168-181 (2012).

23. Acharya, K. R. \& Ackerman, S. J. Eosinophil granule proteins: form and function. J. Biol. Chem. 289, 17406-17415 (2014).

24. Koh, G. C., Shek, L. P., Goh, D. Y., Van Bever, H. \& Koh, D. S. Eosinophil cationic protein: is it useful in asthma? A systematic review. Respiratory Med. 101, 696-705 (2007).

25. Yang, D. et al. Eosinophil-derived neurotoxin (EDN), an antimicrobial protein with chemotactic activities for dendritic cells. Blood 102, 3396-3403 (2003).

26. Torrent, M., Navarro, S., Moussaoui, M., Nogues, M. V. \& Boix, E. Eosinophil cationic protein high-affinity binding to bacteria-wall lipopolysaccharides and peptidoglycans. Biochemistry 47, 3544-3555 (2008).

27. Domachowske, J. B., Bonville, C. A., Dyer, K. D. \& Rosenberg, H. F. Evolution of antiviral activity in the ribonuclease $A$ gene superfamily: evidence for a specific interaction between eosinophil-derived neurotoxin (EDN/RNase 2) and respiratory syncytial virus. Nucleic Acids Res 26, 5327-5332 (1998).

28. Domachowske, J. B., Dyer, K. D., Adams, A. G., Leto, T. L. \& Rosenberg, H. F. Eosinophil cationic protein/RNase 3 is another RNase A-family ribonuclease with direct antiviral activity. Nucleic Acids Res 26, 3358-3363 (1998).

29. Ackerman, S. J., Gleich, G. J., Loegering, D. A., Richardson, B. A. \& Butterworth, A. E. Comparative toxicity of purified human eosinophil granule cationic proteins for schistosomula of Schistosoma mansoni. Am. J. Trop. Med Hyg. 34, 735-745 (1985).

30. Fort, M. M. et al. IL-25 induces IL-4, IL-5, and IL-13 and Th2-associated pathologies in vivo. Immunity 15, 985-995 (2001).

31. Schmitz, J. et al. IL-33, an interleukin-1-like cytokine that signals via the IL-1 receptor-related protein ST2 and induces T helper type 2-associated cytokines. Immunity 23, 479-490 (2005).

32. Rickel, E. A. et al. Identification of functional roles for both IL-17RB and IL-17RA in mediating IL-25-induced activities. J. Immunol. 181, 4299-4310 (2008)

33. Enoksson, M. et al. Intraperitoneal influx of neutrophils in response to IL-33 is mast cell-dependent. Blood 121, 530-536 (2013).

34. Barlow, J. L. et al. IL-33 is more potent than IL-25 in provoking IL-13-producing nuocytes (type 2 innate lymphoid cells) and airway contraction. J. Allergy Clin. Immunol. 132, 933-941 (2013).

35. Yamada, K. J. et al. Eosinophil-associated ribonuclease 11 is a macrophage chemoattractant. J. Biol. Chem. 290, 8863-8875 (2015).

36. Murray, P. J. et al. Macrophage activation and polarization: nomenclature and experimental guidelines. Immunity 41, 14-20 (2014).

37. Wellenstein, M. D. et al. Loss of p53 triggers WNT-dependent systemic inflammation to drive breast cancer metastasis. Nature 572, 538-542 (2019)

38. Cormier, S. A. et al. T(H)2-mediated pulmonary inflammation leads to the differential expression of ribonuclease genes by alveolar macrophages. Am. J. Respir. Cell Mol. Biol. 27, 678-687 (2002).

39. Wilkinson, P. C. Random locomotion; chemotaxis and chemokinesis. A guide to terms defining cell locomotion. Immunol. Today 6, 273-278 (1985).

40. Jablonski, K. A. et al. Novel Markers to Delineate Murine M1 and M2 Macrophages. PLOS ONE 10, e0145342 (2015).

41. Lu, L. et al. Time Series miRNA-mRNA integrated analysis reveals critical miRNAs and targets in macrophage polarization. Sci. Rep. 6, 37446 (2016).

42. Cormier, S. A. et al. Mouse eosinophil-associated ribonucleases: a unique subfamily expressed during hematopoiesis. Mamm. Genome 12, 352-361 (2001).
43. Larson, K. A. et al. Two highly homologous ribonuclease genes expressed in mouse eosinophils identify a larger subgroup of the mammalian ribonuclease superfamily. Proc. Natl Acad. Sci. USA 93, 12370-12375 (1996).

44. Zhang, J., Dyer, K. D. \& Rosenberg, H. F. Evolution of the rodent eosinophilassociated RNase gene family by rapid gene sorting and positive selection. Proc. Natl Acad. Sci. USA 97, 4701-4706 (2000).

45. Panov, K. I. et al. Ribonuclease A mutant His119 Asn: the role of histidine in catalysis. FEBS Lett. 398, 57-60 (1996).

46. Di Valentin, E. et al. New asthma biomarkers: lessons from murine models of acute and chronic asthma. American journal of physiology. Lung Cell. Mol. Physiol. 296, L185-L197 (2009).

47. Louten, J. et al. Biomarkers of disease and treatment in murine and cynomolgus models of chronic asthma. Biomark. Insights 7, 87-104 (2012).

48. Cautivo, K. M. \& Molofsky, A. B. Regulation of metabolic health and adipose tissue function by group 2 innate lymphoid cells. Eur. J. Immunol. 46, 1315-1325 (2016).

49. Saluzzo, S. et al. First-breath-induced type 2 pathways shape the lung immune environment. Cell Rep. 18, 1893-1905 (2017).

50. Molofsky, A. B. et al. Innate lymphoid type 2 cells sustain visceral adipose tissue eosinophils and alternatively activated macrophages. J. Exp. Med. 210, 535-549 (2013).

51. Jenkins, S. J. et al. Local macrophage proliferation, rather than recruitment from the blood, is a signature of TH2 inflammation. Science 332, 1284-1288 (2011).

52. Mantovani, A., Sica, A. \& Locati, M. Macrophage polarization comes of age. Immunity 23, 344-346 (2005).

53. Linehan, S. A. et al. IL-4 receptor signaling is required for mannose receptor expression by macrophages recruited to granulomata but not resident cells in mice infected with Schistosoma mansoni. Lab Investig 83, 1223-1231 (2003).

54. Heller, N. M. et al. Type I IL-4Rs selectively activate IRS-2 to induce target gene expression in macrophages. Sci. Signal 1, ra17 (2008).

55. Liu, H. et al. Alternative splicing analysis in human monocytes and macrophages reveals MBNL1 as major regulator. Nucleic Acids Res 46, 6069-6086 (2018).

56. Chen, F. et al. An essential role for TH2-type responses in limiting acute tissue damage during experimental helminth infection. Nat. Med 18, 260-266 (2012).

57. Bouchery, T. et al. Hookworms evade host immunity by secreting a deoxyribonuclease to degrade neutrophil extracellular traps. Cell Host Microbe 27, 277-289 e276 (2020).

58. Woytschak, J. et al. Type 2 interleukin-4 receptor signaling in neutrophils antagonizes their expansion and migration during infection and inflammation. Immunity 45, 172-184 (2016).

59. Grigolato, F., Egholm, C., Impellizzieri, D., Arosio, P. \& Boyman, O. Establishment of a scalable microfluidic assay for characterization of population-based neutrophil chemotaxis. Allergy. https://doi.org/10.1111/all.14195 (2020).

60. Impellizzieri, D. et al. IL-4 receptor engagement in human neutrophils impairs their migration and extracellular trap formation. J. Allergy Clin. Immunol. 144 267-279 e264 (2019).

61. Egholm, C., Heeb, L. E. M., Impellizzieri, D. \& Boyman, O. The regulatory effects of interleukin-4 receptor signaling on neutrophils in type 2 immune responses. Front Immunol. 10, 2507 (2019).

62. Mould, A. W., Matthaei, K. I., Young, I. G. \& Foster, P. S. Relationship between interleukin-5 and eotaxin in regulating blood and tissue eosinophilia in mice. J. Clin. Investig 99, 1064-1071 (1997).

63. Mishra, A., Hogan, S. P., Lee, J. J., Foster, P. S. \& Rothenberg, M. E. Fundamental signals that regulate eosinophil homing to the gastrointestinal tract. J. Clin. Investig 103, 1719-1727 (1999).

64. Nussbaum, J. C. et al. Type 2 innate lymphoid cells control eosinophil homeostasis. Nature 502, 245-248 (2013).

65. Mayadas, T. N., Cullere, X. \& Lowell, C. A. The multifaceted functions of neutrophils. Annu Rev. Pathol. 9, 181-218 (2014).

Open Access This article is licensed under a Creative Commons Attribution 4.0 International License, which permits use, sharing, adaptation, distribution and reproduction in any medium or format, as long as you give appropriate credit to the original author(s) and the source, provide a link to the Creative Commons license, and indicate if changes were made. The images or other third party material in this article are included in the article's Creative Commons license, unless indicated otherwise in a credit line to the material. If material is not included in the article's Creative Commons license and your intended use is not permitted by statutory regulation or exceeds the permitted use, you will need to obtain permission directly from the copyright holder. To view a copy of this license, visit http://creativecommons. org/licenses/by/4.0/.

(c) The Author(s) 2020 